\title{
Resampling approach for cluster model selection
}

\author{
Z. Volkovich · Z. Barzily • G.-W. Weber • \\ D. Toledano-Kitai $\cdot$ R. Avros
}

Received: 23 February 2010 / Revised: 19 September 2010 / Accepted: 17 January 2011 /

Published online: 18 March 2011

(C) The Author(s) 2011

\begin{abstract}
In cluster analysis, selecting the number of clusters is an "ill-posed" problem of crucial importance. In this paper we propose a re-sampling method for assessing cluster stability. Our model suggests that samples' occurrences in clusters can be considered as realizations of the same random variable in the case of the "true" number of clusters. Thus, similarity between different cluster solutions is measured by means of compound and simple probability metrics. Compound criteria result in validation rules employing the stability content of clusters. Simple probability metrics, in particular those based on kernels, provide more flexible geometrical criteria. We analyze several applications of probability metrics
\end{abstract}

Editors: Süreyya Özögür-Akyüz, Devrim Ünay, and Alex Smola.

Z. Volkovich $(\varangle) \cdot$ Z. Barzily $\cdot$ D. Toledano-Kitai $\cdot$ R. Avros

Ort Braude College of Engineering, Karmiel 21982, Israel

e-mail: vlvolkov@braude.ac.il

Z. Barzily

e-mail: zbarzily@braude.ac.il

D. Toledano-Kitai

e-mail: dvora@braude.ac.il

R. Avros

e-mail: r_avros@braude.ac.il

G.-W. Weber

Institute of Applied Mathematics, Middle East Technical University, 06531 Ankara, Turkey

e-mail: gweber@metu.edu.tr

G.-W. Weber

University of Siegen, Siegen, Germany

G.-W. Weber

University of Aveiro, Aveiro, Portugal

G.-W. Weber

Universiti Teknologi Malaysia, Skudai, Malaysia 
combined with methods intended to simulate cluster occurrences. Numerical experiments are provided to demonstrate and compare the different metrics and simulation approaches.

Keywords Cluster analysis · Clustering · Partitioning · Unsupervised learning · Cluster stability $\cdot$ Two sample test $\cdot$ Kernel based distances

\section{Introduction}

Cluster analysis is one of the main tools in machine learning typically employed in order to identify meaningful groups, named clusters, in the data. It is indirectly assumed that there is an interval similarity among elements of the same cluster. A distortion function which describes dissimilarity between objects is commonly used to measure the differences between data items.

The quality of a partition often formulated as the expected value of the distortion, represents a criterion intended to be minimized. Cluster analysis distinguishes between two types of methods. The first type, called clustering methods, deals with finding of partition in the data elements into a given number of clusters. No additional external information in the clusters process is available. The second type, called validation methods, deals with determining the optimal ("true") number of clusters in a dataset. Here, the clustering solutions are compared according to the predefined rule and the optimal number of clusters is chosen as the one which yields the optimal quality.

The distinction between clustering methods and validation methods as two types of methods in cluster analysis is debatable since clustering methods are usually considered as input to validation methods.

The current paper is devoted to the determination of the true number of clusters. This essential cluster analysis task may have more than one solution and thus it is recognized as "ill posed" (Jain and Dubes 1988; Gordon 1999). For instance, the result can be dependent on the units by which the data is measured (see, for example, Chakravarthy and Ghosh 1996). Although, many approaches were proposed to solve this problem, until now none has been accepted as superior.

From a geometrical points of view, cluster validation has been studied in the papers: Dunn (1974), Hubert and Schultz (1976) (C-index), Calinski and Harabasz (1974), Hartigan (1985), Krzanowski and Lai (1985), Sugar and James (2003), Gordon (1994), Milligan and Cooper (1985) and Tibshirani et al. (2001) (the Gap Statistic method). Another standpoint is based on stability models where pairs of clustered samples from the same data source are compared. Reiterating a clustering algorithm for those pairs enables to measure the variability of clustering solutions. High consistency of the clusters properties is identified with partitions reliability (Cheng and Milligan 1996). The number of clusters that maximizes cluster stability is frequently used to evaluate the "true" number of clusters. In papers by Levine and Domany (2001), by Ben-Hur et al. (2002) and by Ben-Hur and Guyon (2003) stability criteria are understood as the proportions of times that pairs of elements maintain the same membership under a rerun of the clustering algorithm. Mufti et al. (2005) exploit Loevinger's measure of isolation to determine a stability function. Another perspective employs external partition correlation indexes as a stability enormity. For example, this method has been implemented in the known Clest method of Dudoit and Fridlyand (2002). A general prediction based resampling approach has been proposed in Roth et al. (2002), Lange et al. (2003, 2004). Tibshirani and Walther (2005) employ a similar prediction strength procedure. Jain and Moreau (1987) offered the dispersions of empirical distributions as a stability measure. 
Another methodology for the determination of the true number of clusters is provided by nonparametric density estimation. It matches the clusters to the probability distribution function density peaks. Thus, the clustering appoints each item to one "domain of attraction" of the density modes. Evidently, Wishart (1969) firstly offered to look for modes in order to discover the cluster structure.

This idea was offered by Hartigan $(1975$, Sect. 11; 1981) to establish the concept of high density modes clusters. The clusters amount is proposed to be the number of areas, the modes of density of which are bigger than a given threshold. From this standpoint, clusters are imaged as isolated islands of "high" density in the ocean of "low" densities. This concept has been later implemented in numerous papers (see, for example, Cuevas et al. 2000, 2001; Stuetzle 2003).

Goodness of fit test procedures allows another approach. Particularly, Pelleg and Moore (2000) provided, so named, $X$-means algorithm. Using this algorithm, the best score of the so-called Bayesian Information Criterion Kass (1995) is calculated and it detects the true number of clusters. Hamerly and Elkan (2003) apply another scoring criteria in the $G$ means algorithm. Here, a statistical projection procedure is used to test the hypothesis that the data in a cluster drawn from a Gaussian distribution. This algorithm checks an initial small amount of clusters and divides into two clusters the ones which declined as Gaussian.

In the spirit of the $G$-means algorithm, Feng and Hamerly (2006) present an algorithm, called $P G$-means (PG stands for projected Gaussian) which also applies projections to the clustering model. $P G$-means employs the standard Gaussian mixture model with Expectation-Maximization algorithm. Here, any Gaussian mixture based technique can be applied. An Information-Theoretic perspective of the cluster validation problem was discussed by Still and Bialek (2004).

In the papers Volkovich et al. (2008), Barzily et al. (2009) and Volkovich and Barzily (2007) methods using the goodness of fit tests are suggested. The etalon cluster distributions are constructed here, using a model designed to represent well-mixed samples within the clusters.

Volkovich et al. (2009a) use the binomial model of the $K$-Nearest Neighbors belonging to the own point's sample. Another model suggested by Volkovich et al. (2008) and Barzily et al. (2009), focuses on the distances between clustered samples drawn from the clusters cores and the whole population correspondingly.

In the current article we introduce a new general cluster stability methodology which makes it possible to reformulate many existing approaches from a new generic point of view and offer new cluster stability criteria. Clustered samples elements are considered as instances of random variables such that the partition quality is measured by probability metrics between the instances. Our perception suggests a statistical homogeneity, of the clustered samples, in the case of the "true" number of clusters. I.e., we presume that sample occurrences in a cluster appear to be, in this situation, independent realizations of the same random variable.

The proposed point of view conceptually generalizes several previously referred methods. So, the approaches presented in Lange et al. (2002, 2003, 2004) and Tibshirani and Walther (2005) employ Breckenridge's idea (Breckenridge 1989). This approach assesses the cluster solutions resemblance, resting upon a classifier trained by means of a second (clustered) dataset. As usual, the similarity is characterized via closeness offer of clusters contents, as measured, in fact, by the indicator probability metric. On the other hand, the papers of Volkovich et al. (2008), Barzily et al. (2009), Volkovich and Barzily (2007) and Volkovich et al. (2009a) use variants of the goodness of fit tests based on the, so called, "well mingled samples" concept where in the case of the "true" number of clusters samples 
occurrences in clusters are suggested to be mingled to the extent they are mingled in whole population. Obviously, the contents based approaches can also be treated from this standpoint and closeness of samples occurrences can be evaluated by simple probability metrics (two-sample statistics test). The contents based approaches, and the geometrical ones, are actually implementing the same general cluster model. Thus, they can be understood and studied from a uniform point of view. To the best of our knowledge, the offered comprehensible conception is novel in cluster validation problematic. The paper is devoted to the description and design of such a model. Consequently, as it was mentioned earlier, we estimate the "true" number of clusters by examining the stability of samples appearances within the clusters constructed by a clustering algorithm. Although we can not directly select samples from unknown clusters, we rely on the fact that, appropriately clustered samples drawn from whole population can serve as (or simulate) i.i.d. samples, drawn from the clusters. It is proposed to quantify the stability rate via simple or compound probability metrics, such that in the stable partition case, these metrics are expected to be small.

Complicated samples configuration and algorithms weaknesses seriously amplify the noise level of the procedure. To overcome this obstacle, conclusions should be established on a sufficient amount of data and repetitive rerunning of the clustering algorithm. Due to the fact that within clusters distances decrease as the number of clusters increases (see, for example, Lange et al. 2003 and Tibshirani et al. 2001), we use a suitable normalization to balance this result. The "true" number of clusters is chosen as the one which is characterized by the normalized distance empirical distribution mostly concentrated at the origin. Accordingly, the procedure can be generally described as the creation of a normalized empirical metric's distribution for several possible numbers of clusters with the sequential determination of the one most concentrated at zero.

Note, that the clustering solutions interpretation as random variables, is presented in the literature. For example, the issue of the approach offered in Lange et al. (2003), has been formulated in terms of random variables and their characteristics. The idea to use the most concentrated distribution for partitions' similarities, in the true number of clusters case, has been discussed in Ben-Hur et al. (2002).

The rest of the paper is organized in the following form. Section 2 presents the method concept together with basic method components. Particularly, Sect. 2.2 discusses the probability metrics notion and its important examples. In Sect. 2.3 we consider three methods proposed to simulate sample occurrences in clusters. Section 3 reviews kernel based two sample test statistics. Such distances play a very important role in the current methodology because they offer flexible and convenient approaches to construct cluster similarity measures. Section 4 is dedicated to discussion of an algorithmic implantation of the method and numerical experiments provided on an artificial dataset and two real word databases.

\section{The concept}

\subsection{The model}

The main object is a data space $\mathcal{X}$ considered as a finite subset of the Euclidean space $\mathcal{R}^{d}$. We denote by $\langle\mathbf{x}, \mathbf{y}\rangle$ the dot product of two elements $\mathbf{x}, \mathbf{y} \in \mathcal{R}^{d}$ and by $\|\mathbf{x}\|$ the Euclidean norm of $\mathbf{x} \in \mathcal{R}^{d}$. In the paper we use the following notation:

$-\mathbf{R}_{+}=[0,+\infty)$;

$-C_{k}$ is the set $\{1, \ldots, k\}$;

- $\Psi_{k}$ is the set of all possible permutation of the set $C_{k}$; 
- $\mathbf{E}(X)$ is the mathematical expectation value of $X$;

- $\mathcal{P}(\mathcal{X})$ is the set of all probability measures defined on a measurable space $\mathcal{X}$;

- An indicator function $I(A)$ of an event $A$ :

$$
I(A)= \begin{cases}1, & \text { if } A \text { is true } \\ 0, & \text { otherwise }\end{cases}
$$

A partition (clustering)

$$
\Pi_{k}(\mathcal{X})=\left\{\pi_{1}, \ldots, \pi_{k}\right\}
$$

of the space $\mathcal{X}$ is a set consisting of $k$ non-empty clusters such that:

$$
\mathcal{X}=\bigcup_{i=1}^{k} \pi_{i}
$$

and

$$
\pi_{i} \cap \pi_{j}=\emptyset, \quad i \neq j .
$$

For a given partition $\Pi_{k}(\mathcal{X})$, the label function $\alpha\left(\Pi_{k}\right): \mathcal{X} \rightarrow C_{k}$ assigning points to the clusters is defined as

$$
\alpha\left(\Pi_{k}\right)(x)=i, \quad \text { if and only if } \quad x \in \pi_{i}, \quad i=1, \ldots, k,
$$

so

$$
\pi_{i}=\left\{x \in \mathcal{X} \mid \alpha\left(\Pi_{k}\right)(x)=i\right\} .
$$

Each partition leads to the mixed decomposition of the underlying distribution $P_{\mathcal{X}}$ of the set $\mathcal{X}$

$$
P_{\mathcal{X}}=\sum_{i=1}^{k} w_{i} P_{\pi_{i}},
$$

where $w_{i}, i=1, \ldots, k$ are the clusters' probabilities and $P_{\pi_{i}}, i=1, \ldots, k$ are the clusters' distributions. In order to avoid unnecessary additional conditions we furthermore assume that $0<w_{i}, i=1, \ldots, k$.

Let $S$ be a subset of $\mathcal{X}$. A clustering algorithm $\Delta_{k}$ is a function which maps a set $S$ onto $C_{k}$. Obviously, such a function provides a partition of $S$. However, various clusterings, like center-based approaches or spectral clustering, admit natural extensions of the clustering on whole set $\mathcal{X}$. As offered by Strehl and Ghosh (2002), in their cluster ensembles theory terminology, we name such an algorithm a clusterer. We point out that among the input parameters of the algorithm, a source data $S$ and a suggested number of clusters $k$ are included.

A clustering algorithm used for the samples occurrences simulation is an important ingredient of the approach because the partitions constructed by means of the algorithm are intended more or less to reflect the inner hidden data structure, corresponding to the underlying mixture distributions (1). Solutions provided by different algorithms can be essentially different and lead to sufficiently assorted conclusions about stable cluster configurations. To illustrate this we consider partitions in two clusters provided by different algorithms for 

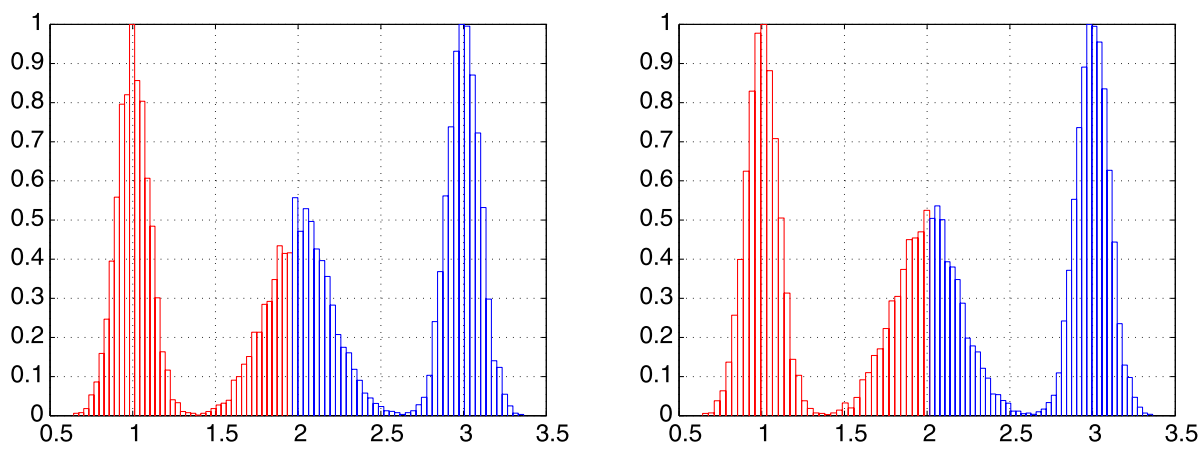

Fig. 1 Outcomes of repeated clusterings by means of the standard $k$-means algorithm
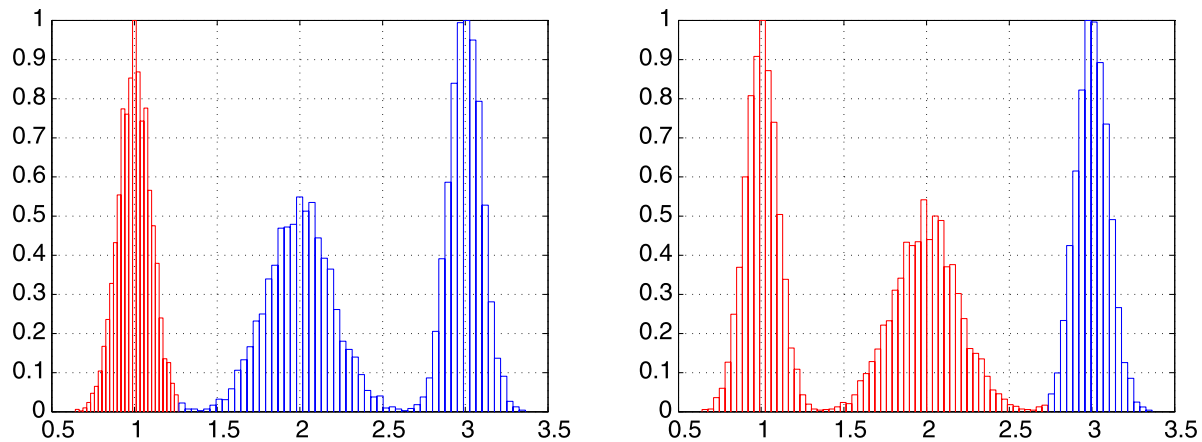

Fig. 2 Outcomes of repeated clusterings by means of the $C E M$ algorithm

a dataset on the real line simulated as a mix of three Gaussian components. In Fig. 1 outcomes of two repeated partitions into 2 clusters of the randomly initialized standard $k$-means algorithm are presented.

Figure 2 demonstrates the result obtained by a version of the known $E M$ algorithm.

As it can be deduced, the $k$-means algorithm recovers a "false" stable two cluster construction. However, a more flexible $E M$ approach hints a possible unsteadiness of the twoclusters structure. Here, we used the Classification $E M$ algorithm (CEM) introduced by Celeux and Govaert (1992) as a version of the classical EM method. Note that the standard $k$-means algorithm can be viewed as its partial case corresponding to the equally-sized spherical clusters.

A meta-algorithm implementing the presented method can be expressed as follows:

\section{Algorithm}

1. Repeat for each tested number of clusters from $k_{\min }$ to $k_{\max }$;

2. Repeat the following steps for pairs randomly drawn without replacement samples;

3. Simulate samples occurrences in the clusters with the help a clustering algorithm;

4. Calculate probability metric values between the occurrences of the samples within the clusters;

5. Calculate a summarizing value representing the distance between the samples in the partition; 
6. Normalize distances values;

7. The estimated number of clusters is the one whose normalized distribution, of the summarizing value, is mostly concentrated at the origin.

\subsection{Probability metrics}

A probability metric is a rule setting dissimilarity amid random variables or vectors. Our intention in this section is to review the probability metrics notion and to discuss several important examples. The probability metrics theory is stated in Zolotarev (1997) and Rachev (1991). Let $\Omega$ denotes a measurable space with $\sigma$-algebra $\mathcal{B}$ and the probability measure $P$. Denote by $\mathcal{D}$ the set of all random variables defined on $(\Omega, \mathcal{B}, P)$. A mapping Dis: $\mathcal{D} \times \mathcal{D} \rightarrow$ $\mathbf{R}_{+}$is said to be a probability metric if for all $X, Y, Z \in \mathcal{D}$ the following conditions are fulfilled:

1. The identity property:

$\operatorname{Dis}(X, Y)=0$ if and only if $X=Y$ almost sure, i.e., $P(X=Y)=1$.

Sometimes it is presented in more weak forms:

a. $\operatorname{Dis}(X, Y)=0$ if $X=Y$ almost surely.

If only this condition holds then the metric is called semi-metric.

b. Dis $(X, Y)=0$ implies equality of the $X$ and $Y$ distributions.

If only this condition holds then the metric is called simple, otherwise the metric is called compound.

2. The symmetry axiom:

$\operatorname{Dis}(X, Y)=\operatorname{Dis}(Y, X)$.

3. The triangle inequality axiom:

$\operatorname{Dis}(X, Y) \leq \operatorname{Dis}(X, Z)+\operatorname{Dis}(Z, Y)$.

\subsubsection{Compound metrics}

Compound distance can be applied as measures of uncertainty. Actually, let $\operatorname{Dis}\left(X, X_{1}\right)$ be a compound distance. For a pair $X, X_{1}$ of i.i.d. variables the equation

$$
\operatorname{Dis}\left(X, X_{1}\right)=0
$$

implies $P\left(X=X_{1}\right)=1$. Explicitly, $X$ is a constant almost surely. By this reason, the value

$$
d(X)=\operatorname{Dis}\left(X, X_{1}\right)
$$

is called an index concentration measure derived by the compound distance Dis.

In the clustering perception two measurable natural spaces $\Omega=C_{k}$ and $\Omega=\mathcal{X} \subset \mathcal{R}^{d}$ endowed with their inherent $\sigma$-algebras become visible. The amount of clusters is typically suggested to be not too large. Consequently, the discrete space $\Omega=C_{k}$ owns the small cardinality property, and all probability metrics defined here are evidently straightly comparable. The most explicable from the clustering point of view metric is the indicator one

$$
\operatorname{Dis}_{0}(X, Y)=P(X \neq Y) \text {. }
$$

The associated concentration measure

$$
d(X)=\operatorname{ess} \sup P\left(X \neq X_{1}\right)
$$


appears, de-facto, in stability based approaches (see, e.g. Dudoit and Fridlyand 2002; Levine and Domany 2001; Lange et al. 2003) as a disagreement measure between repeated solutions given for the same clustered set. A normalization is provided here according to the "worst" distribution assuming the absence of the clustering structure namely, $X$ and $X_{1}$ are uniformly distributed on $C_{k}$. In this case the random variable

$$
Z=I\left(X \neq X_{1}\right)
$$

is obviously a Bernoulli random variable with success probability $\left(1-\frac{1}{k}\right)$, and a normalized version of $d(X)$ is constructed as

$$
\tilde{d}(X)=\frac{d(X)}{E(Z)}=\frac{d(X)}{\left(1-\frac{1}{k}\right)} .
$$

For the sake of trust, it must be noted that the factor $E(Z)$ is often approximated by a simulation according to the finite sample sizes.

\subsubsection{Simple metrics}

Simple metrics are a very acceptable tool in the so named "two-sample problem". It tests the hypothesis whether two samples were drawn from the same population. Namely, let $F$ and $G$ be two unknown distributions and the null hypothesis

$$
H_{0}: F(x)=G(x)
$$

is tested against the general alternative

$$
H_{1}: F(x) \neq G(x) .
$$

Habitually, a simple distance-statistic value is calculated on the drawn samples. The distribution of the distance is given under the null hypothesis. Within all possible values of the statistic, the most extreme evidence against the null hypothesis is chosen. It defines the critical region of the test. The null hypothesis is rejected if the actual distance value falls in the critical region.

The Kolmogorov-Smirnov test, the Cramer-von Mises test, the Friedman's nonparametric ANOVA test and the Wald-Wolfowitz test must be reminded as the classical univariate procedures for this purpose. Information theoretic measures, like the known KullbackLeibler or Jensen-Shannon divergences quantifying the difference between two probability distributions, are, actually, being considered as simple probability metrics.

For the multivariate case many tests have been also derived (Duran 1976; Conover et al. 1981; Friedman and Rafsky 1979; Zech and Aslan 2005; Henze 1988; Baringhaus and Franz 2004; Hall and Tajvidi 2002; Rosenbaum 2005). The multivariate two sample test based kernel statistics introduced in Gretton et al. (2007a, 2007b, 2008a, 2008b) found many notable applications in the machine learning theory. Closely connected to this methodology a kernel method for the two-sample-problem has been independently early proposed in Klebanov (2003). This test is based on a characterization theorem stated in Zinger et al. (1989). Applications of this approach have been discussed in Klebanov et al. (2001) (see also Klebanov 2005). The two-sample energy test described in Zech and Aslan (2005) can also be interpreted in the framework of this methodology. We consider in details kernel based distances in Sect. 3. 

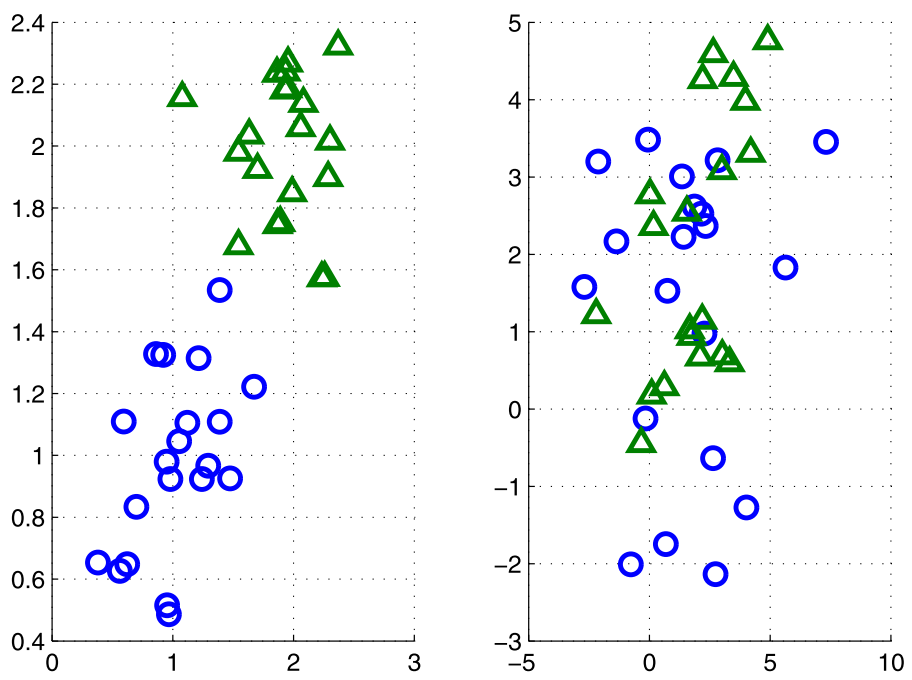

Fig. 3 Elements of two samples mixed in a cluster

As was mentioned earlier, a two sample test statistic (simple probability metric) is intended in the framework of our approach to describe partitions goodness via mingling quality of items belonging to two disjoint i.i.d. samples $S_{1}$ and $S_{2}$ within clusters. To explain this concept let us consider the illustrative examples presented in Figs. 3 and 4. Samples items situated on the left part of the picture are not well mixed amid a cluster; apparently, each sample produces its own cluster. Contrariwise, the samples on the right part are well mingled and define the same stable cluster. We can measure the mixture merit by means of $K$-nearest neighbors fractions of the samples quantified at each point. Obviously, these proportions are approximately equal if the samples are well mixed. Cluster validation has been considered, from this point of view, in the paper Volkovich et al. (2009a). $K$-nearest neighbors type coincidences model applied to this problem in Volkovich et al. (2009b) deals with the statistic:

$$
T_{n, m, k}=\sum_{x \in S_{1} \cup S_{2}} \sum_{r=1}^{K} I(x \text { and } r \text {-th neighbor belong to the same sample), }
$$

which represents the number of all $K$-nearest neighbors type of coincidences. Asymptotic behavior of this statistic has been studied in Henze (1988), and an application to the cluster validation problem considered in Volkovich et al. (2010).

Other acceptable tools in the two sample test problem are based on Minimal Spanning Trees approaches. Figure 4 shows Minimal Spanning Trees constructed inside the cluster presented in Fig. 3. Edges connecting points from different samples are marked by solid red line. The left picture presents only one edge which connects between the items belonging to different clusters. The right picture demonstrates a consistent cluster where the number of the "red" edges is sufficiently big. Thus, a big number of the "red" edges inside a cluster indicates its consistency. The Friedman-Rafsky's test statistic (Friedman and Rafsky 1979), which counts the number of disjoint sub-trees resulting from removing all edges uniting vertices of different samples, quantifies this mixing feature. Cluster stability using this technique has been considered in the papers Barzily et al. (2009), Volkovich et al. (2009b). 

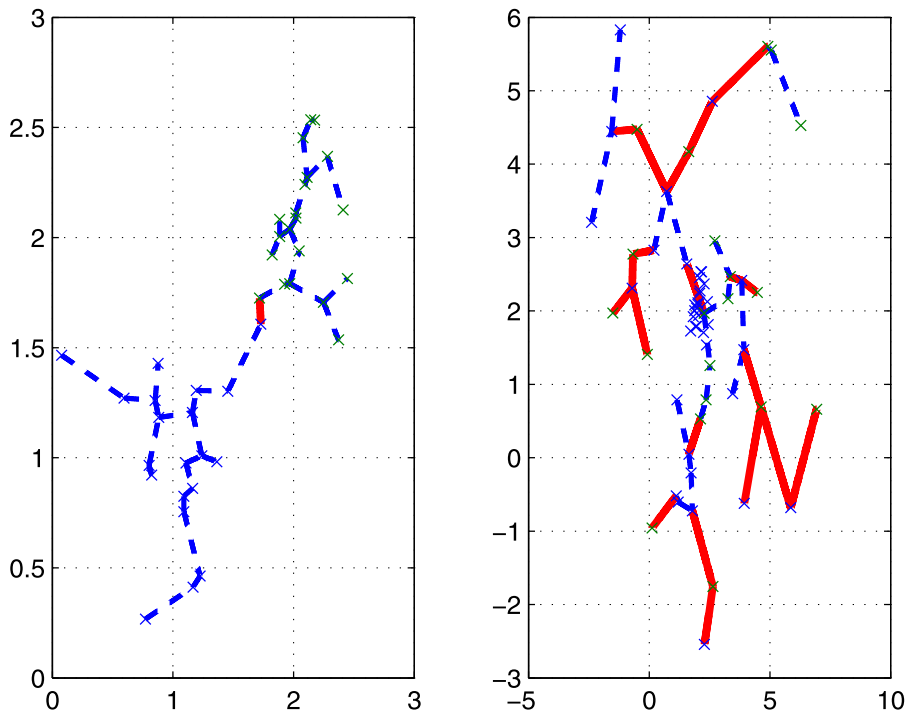

Fig. 4 Examples of minimal spanning trees constructed inside a cluster

\subsubsection{Information metrics}

The Kullback-Leibler divergence (A.K.A. information divergence or relative entropy) is a non-symmetric measure of the difference between probability distributions frequently intuited as a distance metric ( $K L$-distance). The relative entropy is not a true metric, because it is not symmetric and does not satisfy the triangle inequality. However, it is a natural distance function between a "true" probability distribution to a "target" probability distribution. The $K L$-distance of between the probability distributions of random variables on a discrete measurable space is proposed to be

$$
D_{K L}(\mu \| v)=\sum_{i} \mu\left(x_{i}\right) \log _{2}\left(\frac{\mu\left(x_{i}\right)}{v\left(x_{i}\right)}\right) .
$$

The usual convention, based on continuity arguments, is to take $0-\log \frac{0}{q}=0$ for all real $q$ - and $p-\log \frac{p}{0}=\infty$ for all real non-zero value $p$. So, the relative entropy takes values in $[0, \infty]$. The Jensen-Shannon divergence is a symmetrized and smoothed version of the Kullback-Leibler divergence and is defined as

$$
D_{J S}(\mu \| \nu)=\frac{1}{2}\left(D_{K L}(\mu \| \eta)+D_{K L}(v \| \eta)\right),
$$

where $\eta=\frac{1}{2}(\mu+v)$. These measures can be, in general, employed in our methodology although they are not actual probability metrics. Information stability measures are widely used in cluster analysis, since clustering can be viewed as a strategy for data compression by cleaning out irrelevant information via appointing a representative to each group. Lossy compression is provided by transmitting the data to clusters such that the mutual information between the data and clusters' representatives is minimized subject to fixing the expected distortion (see, Rose et al. 1990; Still and Bialek 2004). 
In fact, a clustering stability approach to find the "true" number of clusters has been offered relying on information measures in Pascual et al. (2010). The method is proposed in the framework of the works (Lange et al. 2003; Lange et al. 2004) where the partition quality is characterized by likeness of two partitions: a partition constructed via the transfer by prediction and a partition that was obtained directly. To avoid a simulation step, the mutual information measure between the true data distribution and the data partition distribution, provided the given clustering algorithm, is considered as the stability criterion. The distributions are estimated by means of two samples drawn from the data, and the distribution closeness is, actually, given by the well-mixing of the samples in the clusters.

\subsection{Simulation}

In this section we discuss methods proposed to simulate sample occurrences in clusters. Note, that these variables can take values in cluster applications in $C_{k}=\{1, \ldots, k\}$ or $\mathcal{X}$. Samples elements in the clusters are being created to represent independent random variables. Therefore, unconnected clustering procedure must be applied for each of the samples. It is intended to avoid possible influences of the samples in the clustering process. This task addresses the problem relating to the comparison between two cluster solutions obtained from different (frequently disjoint) sets. A rendering tool must be developed for this purpose. Mechanisms presented in Levine and Domany (2001) and Ben-Hur et al. (2002) propose to operate on datasets intersection. As pointed out in Lange et al. (2003, 2004), such an approach can be biased.

\subsubsection{First simulation method}

As was mentioned earlier, the methodology was employed in Lange et al. (2002, 2003, 2004) and Breckenridge (1989). It offers to evaluate the likeness of clusterings via a classifier trained by means of a second (clustered) dataset. Apart from a basic clusterer $\Delta_{k}(S)$ this approach uses an additional clusterer $\varepsilon_{k}\left(\Pi_{k}\left(S_{0}\right)\right)(S)$ proposed to extend a partition of the set $S_{0}$ to a partition of set $S$.

The occurrences are here constructed on a set $S_{1} \subset \mathcal{X}$ using an auxiliary subset $S_{2} \subset \mathcal{X}$.

- Two solutions

$$
\begin{gathered}
\Pi_{k}^{(1)}\left(S_{1}\right)=\Delta_{k}\left(S_{1}\right), \\
\Pi_{k}\left(S_{2}\right)=\Delta_{k}\left(S_{2}\right)
\end{gathered}
$$

are determined.

- Additional cluster solution for the dataset $S_{1}$ is obtained as

$$
\Pi_{k}^{(2)}\left(S_{1}\right)=\varepsilon_{k}\left(\Pi_{k}\left(S_{2}\right)\right)\left(S_{1}\right)
$$

This partition would be considered as the occurrences of $S_{1}$ elements in the clusters of the partition $\Pi_{k}\left(S_{2}\right)$ if there were a correspondence between the clusters label in two partitions. Actually, the same cluster can be differently labeled in repeated data partitioning. The problem is caused by the inherent symmetry of partitions according to their labels permutations. 
- This ambiguity is being overcome via determination of an optimal labels permutation providing the minimal dissimilarity amid cluster solutions. To solve this problem a discrepancy measure between the partitions is considered:

$$
D_{k}\left(\alpha\left(\Pi_{k}^{(1)}\left(S_{1}\right)\right), \alpha\left(\Pi_{k}^{(2)}\left(S_{1}\right)\right), \psi\right)=\frac{1}{\left|S_{1}\right|} \sum_{x \in S} I\left(\alpha\left(\Pi_{k}^{(1)}\left(S_{1}\right)\right) \neq \psi\left(\alpha\left(\Pi_{k}^{(2)}\left(S_{1}\right)\right),\right.\right.
$$

where $\psi \in \Psi_{k}$. The needed permutation is found as

$$
\psi^{*}=\underset{\psi \in \Psi_{k}}{\arg \min } D_{k}\left(\alpha\left(\Pi_{k}^{(1)}\left(S_{1}\right)\right), \alpha\left(\Pi_{k}^{(2)}\left(S_{1}\right)\right), \psi\right) .
$$

This problem can be expressed as a partial case of the minimum weighed perfect bivariate matching problem. Computational complexity for solving the problem by the well-known Hungarian method (see, Kuhn 1955) is $O\left(\left|S_{1}\right|+k^{3}\right)$. The process needs time $O\left(\left|S_{1}\right|\right)$ for a weight matrix creation and time $O\left(k^{3}\right)$ to perform the matching itself.

Thus, random occurrences are defined in $C_{k}$ as:

$$
\begin{aligned}
& Y_{1}(x)=\alpha\left(\Pi_{k}^{(1)}(x)\right), \quad x \in S_{1}, \\
& Y_{2}(x)=\psi^{*}\left(\alpha\left(\Pi_{k}^{(2)}(x)\right)\right), \quad x \in S_{1} .
\end{aligned}
$$

Accordingly, random occurrences in the clusters can be accepted as

$$
\begin{aligned}
& Z_{1}^{(j)}=\left\{x \in S_{1} \mid \alpha\left(\Pi_{k}^{(1)}(x)\right)=j, j=1, \ldots, k\right\}, \\
& Z_{2}^{(j)}=\left\{x \in S_{2} \mid \psi^{*}\left(\alpha\left(\Pi_{k}^{(2)}(x)\right)\right)=j, j=1, \ldots, k\right\} .
\end{aligned}
$$

A formal disadvantage of this scheme is that in order to "predict the cluster membership" an additional prediction procedure $\varepsilon_{k}$ must be used. Typically, such a procedure is simply defined, for instance, in the case of centroid based or spectral approaches, however this factor may lead to the model indistinctness.

\subsubsection{Second simulation method}

This method has actually been offered in the papers Volkovich et al. $(2008,2009$ a) in order to construct repeated clustering with a relation to geometrical cluster validation. Here, only one clusterer $\Delta_{k}$ is used.

Let $S_{1}$ and $S_{2}$ be two disjoint samples drawn without replacement from the population $\mathcal{X}$.

- We consider

$$
S=S_{1} \cup S_{2}
$$

and construct

$$
\Pi_{k}(S)=\Delta_{k}(S), \quad \Pi_{k}^{(1)}\left(S_{1}\right)=\Delta_{k}\left(S_{1}\right), \quad \Pi_{k}^{(2)}\left(S_{2}\right)=\Delta_{k}\left(S_{2}\right) .
$$

- Consider occurrences of the samples $S_{1}$ and $S_{2}$ in the clusters:

$$
S_{i, j}=\left\{x \in S_{i} \mid \alpha\left(\Pi_{k}^{(i)}\left(S_{i}\right)\right)(x)=j\right\} \quad(i=1,2 ; j=1,2, \ldots, k) .
$$


- Now we need to coordinate the partitions $\Pi_{k}^{(i)}\left(S_{i}\right), i=1,2$. Note, that for each one of the samples $S_{i}, i=1,2$ two cluster solutions $\Pi_{k}\left(S_{i}\right)$ and $\Pi_{k}^{(i)}\left(S_{i}\right)$ are defined. Matchings of $\Pi_{k}^{(i)}\left(S_{i}\right)$ with $\Pi_{k}\left(S_{i}\right), i=1,2$ indirectly provide a matching between $\Pi_{k}^{(i)}\left(S_{i}\right), i=1,2$. Analogously with (4) and (5), we determine for $i=1,2$ permutations

$$
\psi_{i}^{*}=\arg \min _{\psi \in \Psi_{k}} D_{k}\left(\alpha\left(\Pi_{k}\left(S_{i}\right)\right), \alpha\left(\Pi_{k}^{(i)}\left(S_{i}\right)\right), \psi\right), \quad i=1,2 .
$$

Random occurrences defined in $C_{k}$ can be introduced here on $S$ as:

$$
\begin{aligned}
& Y_{1}(x)=\alpha\left(\Pi_{k}(S)(x)\right), \quad x \in S, \\
& Y_{2}(x)=\psi_{i}^{*}\left(\alpha\left(\Pi_{k}^{(i)}(x)\right)\right), \quad x \in S_{i}, i=1,2 .
\end{aligned}
$$

In turn, random occurrences in the clusters are defined as:

$$
\begin{aligned}
& Z_{1}^{(j)}=\left\{x \in S_{1} \mid \psi_{1}^{*}\left(\alpha\left(\Pi_{k}^{(1)}(x)\right)\right)=j, j=1, \ldots, k\right\}, \\
& Z_{2}^{(j)}=\left\{x \in S_{2} \mid \psi_{2}^{*}\left(\alpha\left(\Pi_{k}^{(2)}(x)\right)\right)=j, j=1, \ldots, k\right\} .
\end{aligned}
$$

\subsubsection{Third simulation method}

In the third simulation method occurrences are constructed resting upon two different disjoint samples $S_{1}$ and $S_{2}$ drawn without replacement from the population $\mathcal{X}$ using an auxiliary subset $S_{3} \subset \mathcal{X}$.

- We introduce

$$
S_{1,3}=S_{1} \cup S_{3}, \quad S_{2,3}=S_{2} \cup S_{3}
$$

and construct

$$
\Pi_{k}^{(1,3)}\left(S_{1,3}\right)=\Delta_{k}\left(S_{1,3}\right), \quad \Pi_{k}^{(2,3)}\left(S_{2,3}\right)=\Delta_{k}\left(S_{2,3}\right) .
$$

- Now, there are two partitions of the set $S_{3}$ generated by $\Pi_{k}^{(1,3)}\left(S_{1,3}\right)$ and $\Pi_{k}^{(2,3)}\left(S_{2,3}\right)$, accordingly. Operating similarly to reasonings of the first simulation method we can find a permutation

$$
\psi^{*}=\underset{\psi \in \Psi_{k}}{\arg \min } D_{k}\left(\alpha\left(\Pi_{k}^{(1,3)}\left(S_{1,3}\right)\right), \alpha\left(\Pi_{k}^{(2,3)}\left(S_{2,3}\right)\right), \psi\right)
$$

which provides the maximal matching between these partitions.

Analogously with the first method random occurrences are defined in $C_{k}$ as:

$$
\begin{aligned}
& Y_{1}(x)=\alpha\left(\Pi_{k}^{(1,3)}(x)\right), \quad x \in S_{3}, \\
& Y_{2}(x)=\psi^{*}\left(\alpha\left(\Pi_{k}^{(2,3)}(x)\right)\right), \quad x \in S_{3} .
\end{aligned}
$$

Consecutively, random occurrences in the clusters are defined as:

$$
\begin{aligned}
& Z_{1}^{(j)}=\left\{x \in S_{1} \mid \alpha\left(\Pi_{k}^{(1,3)}(x)\right)=j, j=1, \ldots, k\right\}, \\
& Z_{2}^{(j)}=\left\{x \in S_{2} \mid \psi^{*}\left(\alpha\left(\Pi_{k}^{(2,3)}(x)\right)\right)=j, j=1, \ldots, k\right\} .
\end{aligned}
$$


Note, that this approach can be considered in the spirit of the methods used in Levine and Domany (2001) and Ben-Hur et al. (2002). It operates on the set intersection. Actually, attaching $S_{3}$ in $S_{1,3}$ and $S_{2,3}$ can be deemed as a construction of an appropriate intersection which coincides with $S_{3}$ if sets $S_{i}, i=1,2,3$ are disjoint.

\section{Positive and negative definite kernel based distances}

One of the widespread types of two sample statistics is formed by the following distances between distributions:

$$
D_{K}(P, Q)=\left(E_{X, X^{\prime}}\left(K\left(X, X^{\prime}\right)\right)+E_{Y, Y^{\prime}}\left(K\left(Y, Y^{\prime}\right)\right)-2 E_{X, Y}(K(X, Y))\right)^{\frac{1}{2}},
$$

where $X, Y, X^{\prime}, Y^{\prime}$ are mutually independent random variables such that $X, X^{\prime}$ are drawn according to $P$, and $Y, Y^{\prime}$ are drawn according to $Q . K$ is a kernel function supposed to represent, in the spirit of the kernel trick for distances (Schölkopf 2000), a geometrical similarity amid points in a Euclidean space. From the cluster standpoint such a probability distance appears to be very reasonable. Indeed, if we interpret the random variables as occurrences of samples in clusters then $E_{X, X^{\prime}}\left(K\left(X, X^{\prime}\right)\right)$ estimates a general cluster "diameter" built by means of the first sample. The values $E_{Y, Y^{\prime}}\left(K\left(Y, Y^{\prime}\right)\right)$ and $E_{X, Y}(K(X, Y))$ can be analogously explained. So, the summarizing distance value $\operatorname{Dis}(P, Q)$ constitutes as an error in the found "diameter" estimations and its "small" magnitude hints that the cluster structure can be stable.

In machine learning, kernel methods are being turned recently into a progressively common instrument. The main reason for this popularity is the "kernel trick" proposed in Aizerman et al. (1964). This method is intended to employ a linear algorithm to care of a nonlinear problem so that the patterns are embedded into a higher-dimensional space, where the problem can be solved by means of a linear classifier. The source dataset is primarily fed into feature space

$$
\phi: \mathcal{X} \rightarrow \mathcal{F}, \quad x \mapsto \phi(x),
$$

then evaluated via a dot product

$$
K\left(x, x^{\prime}\right)=\left\langle\phi(x), \phi\left(x^{\prime}\right)\right\rangle .
$$

As usual, a kernel $K$ is chosen without any concrete information according to the mapping $\phi$ in order to shun working in the latent high-dimensional space $\mathcal{F}$. The Mercer's theorem (Schoenberg 1938) asserts that each continuous, symmetric, positive semi-definite kernel function can be represented as a dot product in a high-dimensional space. The success of support vector machines introduced by Cortes and Vapnik (1995) has extended the application of kernels to many learning areas (e.g., Kernel PCA: Schölkopf et al. 1998). Kernel methods are frequently used in clustering applications (see, for example, Ben-Hur et al. 2001; Girolami 2002; Filippone et al. 2008). Encyclopedic accounts of the kernel methods are available at Schölkopf and Smola (2002). Apparently, the most widespread two sample test, in the machine learning area, is a kernel two sample test, introduced in papers Gretton et al. (2007a, 2007b, 2008a, 2008b). 


\subsection{Real positive and negative definite kernels}

First we state several known facts about real positive and negative definite kernels, which can be found, for example, in Schoenberg (1938), Berg et al. (1984), Haussler (1999) and Schölkopf and Smola (2002). Let $\mathcal{X}$ be a finite set.

Definition 1 Let $K$ be a symmetric function on $\mathcal{X} \times \mathcal{X}$, i.e., $K(x, y)=K(y, x)$ for any $x$, $y \in \mathcal{X}$.

- If for any $n \geq 1$ and for any $x_{1}, \ldots, x_{n} \in \mathcal{X}$ the matrix $\mathbf{K}_{i j}=K\left(x_{i}, x_{j}\right)$ is positive definite, i.e.,

$$
\sum_{i, j} \mathbf{K}_{i j} c_{i} c_{j} \geq 0
$$

for any real numbers $c_{1}, \ldots, c_{n}$ then $K$ is called positive definite kernel. If the equality is reached only if $c_{i}=0, i=1, \ldots, n$, then the kernel is called strongly positive definite.

- If for any $n \geq 1$ and for any $x_{1}, \ldots, x_{n} \in \mathcal{X}$

$$
\sum_{i, j} N\left(x_{i}, x_{j}\right) c_{i} c_{j} \leq 0
$$

for any real numbers $c_{1}, \ldots, c_{n}$ such that

$$
\sum_{i=1}^{n} c_{i}=0,
$$

then $N$ is called negative definite kernel. If the equality is reached only if $c_{i}=0, i=$ $1, \ldots, n$, then the kernel is called strongly negative definite.

Note that, the positive definite kernel is often called the Mercer kernels, and the notion "conditionally positive definite kernel" is used instead of the notion "negative definite kernel". Actually, if $N$ is conditionally positive definite kernel then $(-N)$ is a negative definite kernel.

It is well known that

1. A function $N(x, y)$ on $\mathcal{X} \times \mathcal{X}$ is a negative definite kernel if and only if the function $N(x, y)+f(x)+f(y)$ is also a negative definite kernel for any function $f$ on $\mathcal{X}$;

2. Suppose that $N(x, y)$ is a negative definite kernel such that $N(z, z)=0$ for some $z \in \mathcal{X}$ then the function

$$
K(x, y)=N(x, z)+N(z, y)-N(x, y)
$$

is a positive definite kernel.

3. If $K(x, y)$ is a positive definite kernel then the function

$$
N(x, y)=K(x, x)+K(y, y)-2 K(x, y)
$$

is negative definite kernel so that $N(x, x)=0$ for all $x \in \mathcal{X}$.

A connection between the notions of the positive and negative define kernels is also stated as follows: 
Definition 2 Let $K(x, y)$ be a positive definite kernel getting non negative values. The kernel is called infinitely divisible if for each positive integer $n$, there exists a positive definite kernel $K_{n}$ such that $K=K_{n}^{n}$.

The following statements are equivalent:

- $K(x, y)=\exp (-t N(x, y))$ is positive definite kernel for all $t>0$;

$-N(x, y)$ is negative definite kernel.

For the set $\mathcal{X}$ having a linear structure, translation invariant positive definite kernels of the type $K(x, y)=f(x-y)$ are quite widespread in applications. Here, $f$ is named positive definite function. If $\mathcal{X}$ is the Euclidean space $\mathcal{R}^{d}$, then according to the well known Bochner's theorem (see, for example, Lukacs 1970) $f$ is a continuous positive definite function satisfying $f(o)=1$ if and only if $f$ is a characteristic function (the Fourier transform) of a symmetric distribution on $\mathcal{R}^{d}$. In the kernel terminology a symmetric distribution $Q$ is infinitely divisible if the kernel $K(x, y)=g(x-y, Q)$ is positive definite. Here, $g(x, Q)$ is the characteristic function of $Q$. In this case

$$
\lambda(x)=-\log (g(x, Q))=-\int \frac{(\cos (i\langle t \cdot x\rangle)-1)}{\|t\|^{2}} \mu(d t),
$$

where $\mu$ is a finite symmetric measure on $\mathcal{R}^{d}$ (see, for example, Lukacs 1970; Sato 1999). The kernel $N(x, y)=\lambda(x-y)$ is negative definite (see, for example, Lukacs 1970). Particularly, the functions of the type $\psi(x-y)=\|x-y\|^{r}$ produce negative definite kernels generated by the logarithms of the characteristic functions of the, so named, symmetric stable distributions. These functions are strongly negative definite for $0<r<2$. The case $r=2$ corresponds to the symmetric Gaussian laws. Equation (8) is known, in probability theory, as the Levy-Khintchine representation. Generalizations of this statement have been considered, for example, in Volkovich et al. (2005).

A simple intuitive way to build a feature map satisfying (7) is based on the following theorem (see Aronszajn 1950).

Theorem 1 Let $K(x, y)$ be a positive definite kernel. Then there exists unique Hilbert space $\mathcal{F}=\boldsymbol{H}(K)$ having real functions as elements defined on $\mathcal{X}$ such that

$-k_{y}(\cdot)=K(\cdot, y) \in \boldsymbol{H}(K)$ for all $y \in \mathcal{X}$

- for each $y \in \mathcal{X}$ and $\phi \in \boldsymbol{H}(K)$ the relationship

$$
\left\langle\phi, k_{y}(\cdot)\right\rangle=\phi(y)
$$

is held.

Resting upon this theorem we can create a feature map as

$$
\phi: \mathcal{X} \rightarrow \boldsymbol{H}(K), \quad x \longmapsto k_{x}(\cdot) .
$$

In particular, according to definition of the dot product we have

$$
\left\langle k_{x}(\cdot), k_{y}(\cdot)\right\rangle=K(x, y)
$$

for all $x, y \in \mathcal{X}$. So, positive definite kernels appear to be nonlinear generalizations of the inherent similarity measure granted by the dot product. The space $\boldsymbol{H}(K)$ is called a Reproducing Kernel Hilbert Space (RKHS).

By a comparable procedure a generalization of dissimilarity measures can be provided. 
Theorem 2 Let $N(x, y)$ be a negative define kernel satisfying $K(x, x)=0$ for all $x \in \mathcal{X}$. Then there exists a Hilbert space $\boldsymbol{H}$ of real valued functions on $\mathcal{X}$ and a mapping $\phi: \mathcal{X}$ $\rightarrow \boldsymbol{H}$ such that

$$
\|\phi(x)-\phi(y)\|^{2}=N(x, y) .
$$

This theorem has been used as a starting point for the kernel trick for distances in machine learning described in Schölkopf (2000). An analog to Theorem 1 generating Theorem 2 has been offers by Schoenberg (1938).

Theorem 3 Let $(\mathcal{X} ; d)$ be a metric space. $(\mathcal{X} ; d)$ is isometric to a subset of Hilbert space if and only if $d^{2}$ is negative definite kernel.

\subsection{Two sample test kernel distances}

\subsubsection{Positive define kernels based distances}

The central idea of kernel based two sample testes introduced in Gretton (2007a, 2007b, 2008a, 2008b) is to use as the distance amid the distribution the maximum mean discrepancy $(M M D)$ calculated between two distributions' images in an appropriate RKHS. Let us consider an RKHS $\boldsymbol{H}(K)$ on the separable metric space $\mathcal{X}$ with a continuous feature mapping $\phi(x)$ for all $x \in \mathcal{X}$ and a bounded kernel $K$. A mapping

$$
\mu: \mathcal{P}(\mathcal{X}) \rightarrow \boldsymbol{H}(K)
$$

is defined as the expectation of $\phi(x)$ with respect to the mapped probability measure:

$$
\mu(P)=\int \phi(x) P(d x) .
$$

Consequently, $M M D$ is a distance between two such mappings:

$$
\begin{aligned}
M_{K}(P, Q):= & \|\mu(P)-\mu(Q)\| \\
= & \iint\left\langle\phi\left(x_{1}\right), \phi\left(x_{2}\right)\right\rangle P\left(d x_{1}\right) P\left(d x_{2}\right)+\iint\left\langle\phi\left(y_{1}\right), \phi\left(y_{2}\right)\right\rangle Q\left(d y_{1}\right) Q\left(d y_{2}\right) \\
& -2 \iint\langle\phi(x), \phi(y)\rangle P(d x) P(d y) .
\end{aligned}
$$

Obviously, it can be represented in the form of (4). Moreover, this quantity satisfies all the metric properties excluding the identity quality: the relationship $\operatorname{MMD}_{K}(P, Q)=0$ does not imply $P=Q$. However, this measure is a metric if the kernel is characteristic. It means that $\boldsymbol{H}(K)$ is built on a compact metric space, and the kernel is dense with respect to the $L_{\infty}$ norm in the space of bounded continuous functions defined on this space. As was demonstrated in Steinwart (2001), the Gaussian and the Laplace kernels are universal.

Let us suppose we have two i.i.d. samples $\mathbf{X}=\left\{X_{i}, i=1, \ldots, n\right\}$ and $\mathbf{Y}=\left\{Y_{j}, j=\right.$ $1, \ldots, m\}$ drawn respectably from $P$ and $Q$. A biased empirical estimate of the $M M D$ is

$$
M M D_{K}(\mathbf{X}, \mathbf{Y})=\left[\frac{1}{n^{2}} \sum_{i, j=1}^{n} K\left(X_{i}, X_{j}\right)+\frac{1}{m^{2}} \sum_{i, j=1}^{m} K\left(Y_{i}, Y_{j}\right)-\frac{2}{n m} \sum_{i, j=1}^{n, m} K\left(X_{i}, Y_{j}\right)\right]^{\frac{1}{2}} .
$$


Accordingly, when $n=m$ an unbiased estimate of $M M D$ is the one-sample $U$-statistic

$$
M M D_{K}(\mathbf{X}, \mathbf{Y})=\frac{1}{n(n-1)} \sum_{i \neq j}^{n} h\left(Z_{i}, Z_{j}\right)
$$

where $Z_{i}:=\left(X_{i}, Y_{i}\right)$ and

$$
h\left(Z_{i}, Z_{j}\right):=K\left(X_{i}, X_{j}\right)+K\left(Y_{i}, Y_{j}\right)-K\left(X_{i}, Y_{j}\right)-K\left(Y_{j}, Y_{i}\right)
$$

\subsubsection{Negative define kernels based distances}

The approach outlined in the previous section can be considered in the framework of the slightly more general construction, based on the negative define kernels. The idea of such a method is based on the characterization theorems stated in Zinger et al. (1989) and Klebanov (2003). Several applications of this methodology are presented in Klebanov et al. (2001) and Belopolskaya et al. (2005) while a general clarification can be found in the book (Klebanov 2005).

Let $(\mathcal{X} ; d)$ be a metric space. Suppose that $N$ is real continuous function on $\mathcal{X} \times \mathcal{X}$, and denote by $\mathcal{P}_{N}(\mathcal{X})$ the set of all probability measures satisfying the relationship:

$$
\int|N(x, x)| P(d x)<\infty .
$$

Theorem 4 Let $N$ be a real symmetric continuous function on $\mathcal{X} \times \mathcal{X}$. The inequality

$$
\begin{aligned}
\mathcal{N}(P, Q)= & 2 \iint N(x, y) P(d x) Q(d y)-\iint N(x, y) P(d x) P(d y) \\
& -\iint N(x, y) Q(d x) Q(d y) \geq 0
\end{aligned}
$$

holds for all $P, Q \in \mathcal{P}_{N}(\mathcal{X})$ if and only if $N$ is negative definite kernel. Equality is obtained, in the case where $P=Q$, if and only if, $N$ is strongly negative definite kernel.

The proof is very simple. Let $W \in \mathcal{P}_{N}(\mathcal{X})$ be an arbitrary measure dominating $P$ and $Q$. Denote

$$
H=\frac{d P}{d W}-\frac{d Q}{d W}
$$

So, the inequality (9) can be rewritten in the form

$$
\iint N(x, y) H(x) H(y) W(d x) W(d y) \leq 0 .
$$

The measure $W$ and the function $H$ with zero mean are arbitrary because the measures $P$, $Q$ are arbitrary chosen. Thus, (9) and (10) are equivalent. However, (10) is the same as the definition of negative definite kernel. Further, let us take a negative definite kernel $N(x, y)$ such that $N(x ; x)=0$ for all $x \in \mathcal{X}$. According to the earlier stated properties, of the negative definite kernels, the kernel

$$
K(x, y)=N(x, z)+N(z, y)-N(x, y)
$$


is a positive definite kernel for all $z \in \mathcal{X}$. So,

$$
\mathcal{N}(\mu, v)=\mathcal{R}(\mu, \mu)+\mathcal{R}(v, v)-2 \mathcal{R}(\mu, v),
$$

where

$$
\mathcal{L}(\mu, v)=\iint K(x, y) \mu(d x) v(d y) .
$$

For two independent random variables $X, Y$ with distribution functions $P, Q \in \mathcal{P}_{N}(\mathcal{X})$ the distance $\mathcal{N}$ is represented as

$$
\mathcal{L}(P, Q)=\left(2 \mathbf{E}(N(X, Y))-\mathbf{E}\left(N\left(X, X^{\prime}\right)\right)-\mathbf{E}\left(N\left(Y, Y^{\prime}\right)\right)\right)^{\frac{1}{2}},
$$

where $X^{\prime}, Y^{\prime}$ are independent copies of $X, Y$ and all random variables $X, Y, X^{\prime}, Y^{\prime}$ are mutually independent.

Theorem 5 Let $N$ be strongly symmetric negative definite kernel, satisfying $N(x ; x)=0$ for all $x \in \mathcal{X}$. Then $\mathcal{R}=\mathcal{L}(P, Q)$ is a distance on $\mathcal{P}_{N}(\mathcal{X})$.

Assume now that $(\mathcal{X} ; d)$ is a metric space such that $d^{2}$ is a strongly negative definite kernel. As implied from the theorem, $\mathcal{R}=\mathcal{L}(P, Q)$ is in this case a strictly negative define kernel on $\mathcal{P}_{N}(\mathcal{X})$. According to Theorem 3, the metric space $\left(\mathcal{P}_{N}(\mathcal{X}), \mathcal{R}\right)$ is isometric to a subset of the Hilbert space. Set $\mathcal{X}$ is naturally identified with subset of the point measures in $\mathcal{P}_{N}(\mathcal{X})$, and the considered distance can be described analogously with $M M D$ as the distance between the corresponding barycentres in a Hilbert space.

As we have seen, if $K(x, y)$ is a positive definite kernel then the kernel $N(x, y)=$ $K(x, x)+K(y, y)-2 K(x, y)$ is a negative definite one. So, earlier described MMD metrics based construction, could be formally embedded into the negative define kernels based distances scheme, presented in this section.

\subsubsection{Parzen window based distances}

Another kind of kernels commonly used is the Parzen kernels. Parzen windowing is a density estimation technique based on approximations by means of kernel-functions mixtures such that analytical properties of the estimator are inherited from the appropriate kernel properties (Parzen 1962). A suitable Parzen window typically demonstrates the following properties (Wand and Jones 1995):

- It is unimodal and symmetric;

- It is non-negative;

- It integrates to one.

As usual, it is not essential to operate with a window integrates to one. Supposedly, it could integrate to a constant, while the scalar factor is easy eliminated in the estimation process. Parzen windowing is also known as a kernel density estimation method. For a i.i.d. sample $\left\{X_{i}, i=1, \ldots, n\right\}$, drawn from the true density $f(x)$, the Parzen window estimate for this density is given as

$$
\widehat{f}(\mathbf{x})=\sum_{i=1}^{n} \psi_{\sigma}\left(\mathbf{x}, X_{i}\right),
$$


where $\psi_{\sigma}(\cdot, \cdot)$ is the Parzen window, a scale parameter $\sigma$ governs the width of the window. In clustering such estimations can arise, for example, in information based clustering approaches (Gokcay and Principe 2002) and also in attempts to estimate the divergence, or "distance", between the overall and a cluster density (Robert and Torbjorn 2008). However, in the cluster validation context it naturally appears to consider the divergence between two cluster density estimation obtained resting upon two samples "drawn" (or simulated) within the cluster:

$$
D_{i}=\int\left(p_{i}^{(1)}(\mathbf{x})-p_{i}^{(2)}(\mathbf{x})\right)^{2} d \mathbf{x}, \quad i=1, \ldots, k .
$$

It is easy to see that

$$
D_{i}=V\left(p_{i}^{(1)}\right)+V\left(p_{i}^{(2)}\right)-2 V\left(p_{i}^{(1)}, p_{i}^{(2)}\right)
$$

where $V\left(p_{i}^{(1)}, p_{i}^{(2)}\right)$ is a "cross information potential"

$$
V\left(p_{i}^{(1)}, p_{i}^{(2)}\right)=\int p_{i}^{(1)}(\mathbf{x}) p_{i}^{(2)}(\mathbf{x}) d \mathbf{x}
$$

The quantity

$$
V(f)=\int f^{2}(x) d x
$$

is often named the information potential (see, e.g. Principe et al. 2002). It is closely related to the Renyi's quadratic entropy, which is linked by the density function $f(x)$ :

$$
H(f)=-\log \int f^{2}(x) d x .
$$

Let us suppose that the densities $p_{i}^{(1)}, p_{i}^{(2)}$ are estimated based on two samples $S^{(1)}, S^{(2)}$ "drawn" from the cluster " $i$ " by means of a Parzen window $\phi_{\sigma}(\cdot, \cdot)$. It is natural to suppose that the divergence value is small for stable clusters. Surly, we have to simulate these samples as was described in Sect. 2.3

$$
\widehat{p}_{i}^{(1)}(\mathbf{x})=\frac{1}{n_{1}} \sum_{x_{j} \in S^{(1)}} \psi_{\sigma}\left(x, x_{j}\right), \widehat{p}_{i}^{(2)}(x)=\frac{1}{n_{2}} \sum_{x_{j} \in S^{(2)}} \psi_{\sigma}\left(x, x_{j}\right),
$$

where $n_{j}=\left|S^{(j)}\right|, j=1,2$. Hence, we obtain the following estimator for $D_{i}$

$$
\begin{aligned}
\widehat{D}_{i}= & \frac{1}{\left(n_{1}\right)^{2}} \sum_{x_{j_{1}} \in S^{(1)}} \sum_{x_{j_{2}} \in S^{(2)}} \psi_{\sigma}\left(x_{j_{1}}, x_{j_{2}}\right)+\frac{1}{\left(n_{2}\right)^{2}} \sum_{x_{j_{1}} \in S^{(1)}} \sum_{x_{j_{2}} \in S^{(2)}} \psi_{\sigma}\left(x_{j_{1}}, x_{j_{2}}\right) \\
& -\frac{2}{n_{1} n_{2}} \sum_{x_{j_{1}} \in S^{(1)}} \sum_{\mathbf{x}_{j_{2}} \in S^{(2)}} \psi_{\sigma}\left(x_{j_{1}}, x_{j_{2}}\right) .
\end{aligned}
$$

This expression coincidences with the Parzen window-based statistic introduced in Anderson et al. (1994) while $\psi_{\sigma}$ is chosen as a translation invariant Mercer kernel

$$
\psi_{\sigma}(x, y)=k\left(\frac{x-y}{\sigma}\right)
$$


We have seen that the Parzen window based distances of type (13) can be simply interpreted from the cluster stability standpoint. However, consistency of such estimations entails the kernel size $\sigma$ to diminish under an increasing sample size. This fact yields that the corresponding two-sample test converges slowly. Furthermore, the MMD based tests demonstrate more admirable performance (Gretton et al. 2008b).

\subsection{The Two-Sample Energy test}

The Two-Sample Energy test for the two-sample problem (Zech and Aslan 2005) considers two i.i.d. samples, $\mathbf{X}=\left\{X_{i}, i=1, \ldots, n\right\}$ and $\mathbf{Y}=\left\{Y_{j}, j=1, \ldots, m\right\}$. The elements of the first sample are considered to have positive charges equaling to $\frac{1}{n}$ and the elements of the second sample are considered to have negative charges equaling to $\left(-\frac{1}{m}\right)$. These charges are providing a total charge of each sample equaling to 1 . According to the one-over-distance law in the electrostatic, it can be concluded that if the samples have the same distribution then the total energy of the united sample asymptotically neglects.

Let us denote by $\Phi_{X, R}$ the energy of a charged sample $X$. This value is calculated in the following way:

$$
\Phi_{\mathbf{X}, R}=\frac{1}{n^{2}} \sum_{i<j}^{n} R\left(\left|X_{i}-X_{j}\right|\right), \quad \Phi_{Y, R}=\frac{1}{m^{2}} \sum_{i<j}^{m} R\left(\left|Y_{i}-Y_{j}\right|\right),
$$

where the function $R$ is suggested to be a continuous, monotonic decreasing function of the Euclidean distance between the charges. Correspondingly, the intersection energy of two samples $X$ and $Y$ is:

$$
\Phi_{X, \mathbf{Y}, R}=-\frac{1}{n m} \sum_{i=1}^{n} \sum_{j=1}^{m} R\left(\left|X_{i}-Y_{j}\right|\right) .
$$

Respectively, the test statistic $\Psi_{X, Y, R}$ is defined as:

$$
\Psi_{\mathbf{X}, \mathbf{Y}, R}=\Phi_{\mathbf{X}, R}+\Phi_{\mathbf{Y}, R}+\Phi_{\mathbf{X}, \mathbf{Y}, R}
$$

Common functions for $R$ are:

$-R(r)=-\ln (r)$

$-R(r)=\frac{1}{r^{\alpha}}, \alpha>0$

$-R(r)=e^{-r^{\alpha}}, \alpha>0$.

The choice $R(r)=-\ln (r)$ ensures that the test is scale invariant. Although equation (14) is obtained resting upon physical considerations, opposed to the ones used in the previous sections, this equation evidently coincidences with the general expression (6).

\section{Numerical experiments}

\subsection{Comments regarding the algorithm}

The algorithm describes the approach in a general form. Like in many kernel-based classification methods, the effectiveness lies principally in the metric selection which is dependent on our intention to deal with contents or with geometrical validation criteria. Correspondingly, geometrical, especially kernel-based, distances must be chosen according to our intuition to reflect inner data structure. Usually, this task can not be carried out according to 
a theoretical procedure. The final decision is commonly selected by combining results obtained for several distance-like functions. In information theoretical approaches (see, Rose et al. 1990, 1993; Tishby et al. 2000; Still and Bialek 2004) the "true" number of clusters is given by means of the tradeoff between the compression and the information rates where the tradeoff parameter is associated with the free energy or the system temperature. However, this elegant method is rarely used for justification of solutions obtained by a procedure based on different perceptions. This is caused by the problems raising in incorporating them into the approach without any information concerning the parameters (mainly, the temperature value).

In the framework of our method and the other approaches testing the goodness of partitions for the possible number of clusters belonging to a given interval (see, for example, Roth et al. 2002; Lange et al. 2003, 2004; Tibshirani and Walther 2005 and Dudoit and Fridlyand 2002) the tradeoff between the compression and the information rates can be expressed by the interval selection. Essentially, this area, $\left[k_{\min }, k_{\max }\right]$ in the algorithm presented in Sect. 2.1, is intended to reflect our predilection according to the true number of clusters in the set; accordingly, the solution obtained can be accepted only as the best one in the area being investigated.

We consider numerical examples for relatively small dimensionality data containing not necessarily linearly-separated clusters. As it was mentioned, the major aim is to place the data into a high-dimensional space, where the problem can be solved by means of a linear classifier. Note, that the comparable methods are commonly demonstrated by examples dealing with collections having depicted configuration.

To evaluate the overall computational complexity of the algorithm we denote

$-K^{*}$ : maximal number of clusters to be tested;

- $J$ : number of pairs of the drawn sample;

- $m$ : size of samples.

It is easy to see that the complexity is bounded by

$$
\begin{aligned}
& \Psi\left(K^{*}, J, m, \operatorname{SM}\left(m, K^{*}\right)\right) \\
& \quad=O\left(K^{*} * J *\left(S M\left(m, K^{*}\right)+D\left(m, K^{*}\right)\right)\right)+O\left(K^{*} * N(J)\right)+O\left(A S\left(K^{*}\right)\right),
\end{aligned}
$$

where

- $S M\left(m, K^{*}\right)$ is the complexity of the simulation step;

- $D\left(m, K^{*}\right)$ is the complexity of calculation of the distance's values within the $K^{*}$ clusters;

- $N(J)$ is the complexity of the normalization step;

- $A S\left(K^{*}\right)$ is the complexity of the calculation of a concentration at the origin index.

The last two parameters are negligible compared to the others. The simulation complexity depends on the clustering algorithm complexity $C\left(m, K^{*}\right)$, and on the complexity of the clusterer $C_{1}\left(m, K^{*}\right)$ involved in the first simulation step. So

- For the first simulation step we have

$$
S M\left(m, K^{*}\right)=O\left(C_{1}\left(m, K^{*}\right)\right)+O\left(C\left(m, K^{*}\right)\right)+O\left(m+K^{* 3}\right) .
$$

The most common situation is when the same algorithm is used for the auxiliary subset clustering with appropriate assignment of the items to the previously obtained clusters (in the auxiliary subset). In this case

$$
C_{1}\left(m, K^{*}\right)=C\left(m, K^{*}\right)+O\left(m * K^{*}\right) .
$$


Accordingly, $O\left(m+K^{* 3}\right)$ is the complexity of the Hungarian method.

- For the second simulation method we have

$$
S M\left(m, K^{*}\right)=O\left(C\left(2 m, K^{*}\right)\right)+O\left(m+K^{* 3}\right) ;
$$

- For the third simulation method we have

$$
S M\left(m, K^{*}\right)=O\left(C\left(m+m_{1}, K^{*}\right)\right)+O\left(m_{1}+K^{* 3}\right) ;
$$

where $m_{1}$ is the size of the auxiliary subset.

In the case of the classical $k$-means algorithm

$$
C\left(m, K^{*}\right)=O\left(m K^{*}\right) .
$$

If the Partitioning Around Medoids (PAM) algorithm (see, Kaufman and Rousseeuw 1990) is employed then

$$
C\left(m, K^{*}\right)=O\left(m^{2} K^{*}\right) .
$$

The PAM algorithm tends to be more robust than the $k$-means algorithm. Similarly, $D\left(m, K^{*}\right)$ is dependent on the probability distance choice. If the MTS Friedman-Rafsky's test statistic (Friedman and Rafsky 1979) is used then $D\left(m, K^{*}\right)$ is bounded by $O\left(m^{2}\right)$. This bound can be also obtained for the kernel based distances discussed in Sect. 3.

\subsection{Algorithm implementation}

In order to compare the ability of the described simulation method and the appropriate probability metrics we provide various numerical experiments on synthetic and real datasets. The concentration distances (2) and (3) have been weighed against kernel based distances of the type (6). It should be noted that, in practice, a problem to estimate the expectation value over partitions obtained for different pairs of samples appears. At this point the cluster coordination problem together with unsteady obstacles of clustering algorithms must be overcome. A natural way to manage it is to operate with the values

$$
\begin{aligned}
D_{j}= & \widehat{M M D}_{K, j}\left(S_{j}^{(1)}, S_{j}^{(2)}\right) \\
= & a b s\left(\frac{1}{\left|S_{j}^{(1)}\right|^{2}} \sum_{X_{i_{1}}, X_{i_{2}} \in S_{j}^{(1)}} K\left(X_{i_{1}}, X_{i_{2}}\right)+\frac{1}{\left|S_{j}^{(2)}\right|^{2}} \sum_{Y_{i_{1}}, Y_{i_{2}} \in S_{j}^{(2)}} K\left(Y_{i_{1}}, Y_{i_{2}}\right)\right. \\
& \left.-\frac{2}{\left|S_{j}^{(1)}\right|\left|S_{j}^{(2)}\right|} \sum_{X_{i_{1}} \in S_{j}^{(1)}, Y_{i_{2}} \in S_{j}^{(2)}} K\left(X_{i_{1}}, Y_{i_{2}}\right)\right)
\end{aligned}
$$

representing distances between simulated samples occurrences $S_{j}^{(1)}$ and $S_{j}^{(2)}$ inside the clusters $j=1, \ldots, k$. To describe whole partition quality these values have to be summarized, for instance, in a such a form:

$$
D_{0}=\max _{j}\left(D_{j}\right),
$$

which characterizes the partition by its "worst" cluster. It is natural to expect that distribution of these values constructed based sufficient amount of drawn samples pairs would be 

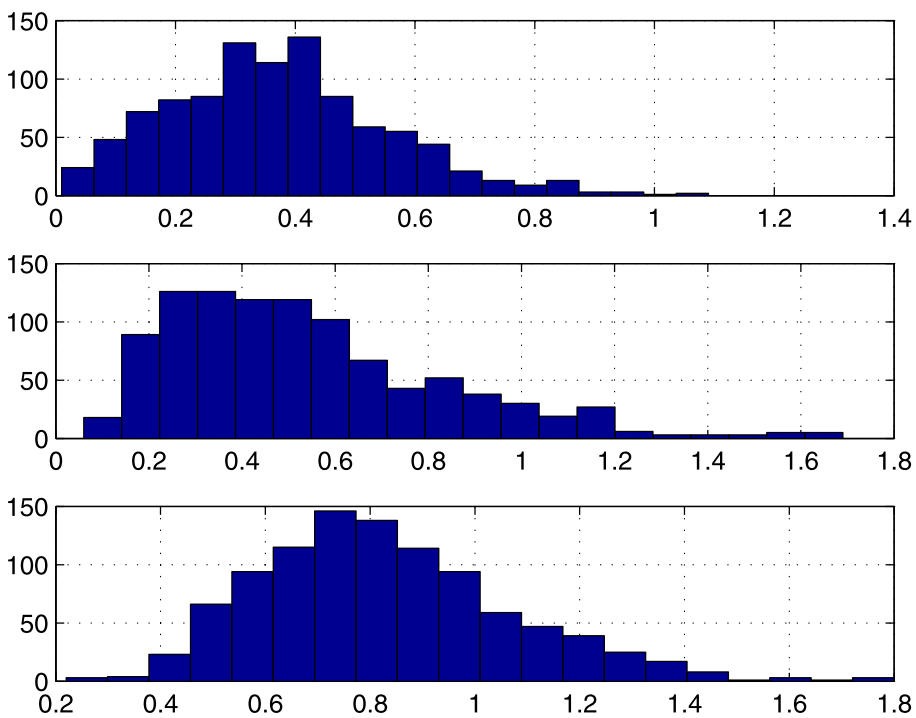

Fig. 5 Examples of the indicator metric histograms

most concentrated at the origin in the case of the "true" number of clusters. This property can be established by many characteristics like empirical normalized mean or percentiles (see, for example, Lange et al. 2003; Volkovich et al. 2008). Here we offer to use another method, which deals with the distance's non-overlapping intervals of the same size. Simply, we divide the whole area of the obtained distance values into numerous intervals having the same size. The relative frequency of the lowest category is the proposed indicator. Its biggest value is intended to indicate the true number of clusters. Obviously, the intervals quantity choice is very essential in this approach. Examples of concentration measure (2) histograms built for the same data, posing source partition into 3 well separated groups, for number clusters 2, 3 and 4 are presented in Figs. 5 and 6.

For the indicator metric given in Fig. 5 the histograms are not locating around the origin and the "true" number of clusters is depicted by the "shortest" right tail. Such an asymmetry is pointed out by relatively small number of categories (10 in our experiments). Another situation arises while kernel based distances are considered. Figure 6 demonstrates histograms of a kernel distance found for the same data. In this case many inherently small distance values are created. In order to focus the "true" number of clusters characterized by preferred amount of these values, the number of categories for histograms has to be sufficiently large (30 in our experiments).

In our experiments the samples are clustered by the standard $k$-means algorithm. All calculations are repeated 10 times in order to examine their persistence and represented via error-bar plots, sized one standard deviation. Note, that the concentrated measures (2) and (3) combined with the first simulation approach and appropriate normalization, coincidences with the cluster stability method offered in Lange et al. (2002, 2003, 2004). Thus, for this metric we present the normalized proportions' means $\left(m_{i}, i=1,2,3\right)$, as was proposed in these articles, together with the suitable frequencies of the lowest groups $\left(f_{i}, i=1,2,3\right)$. 

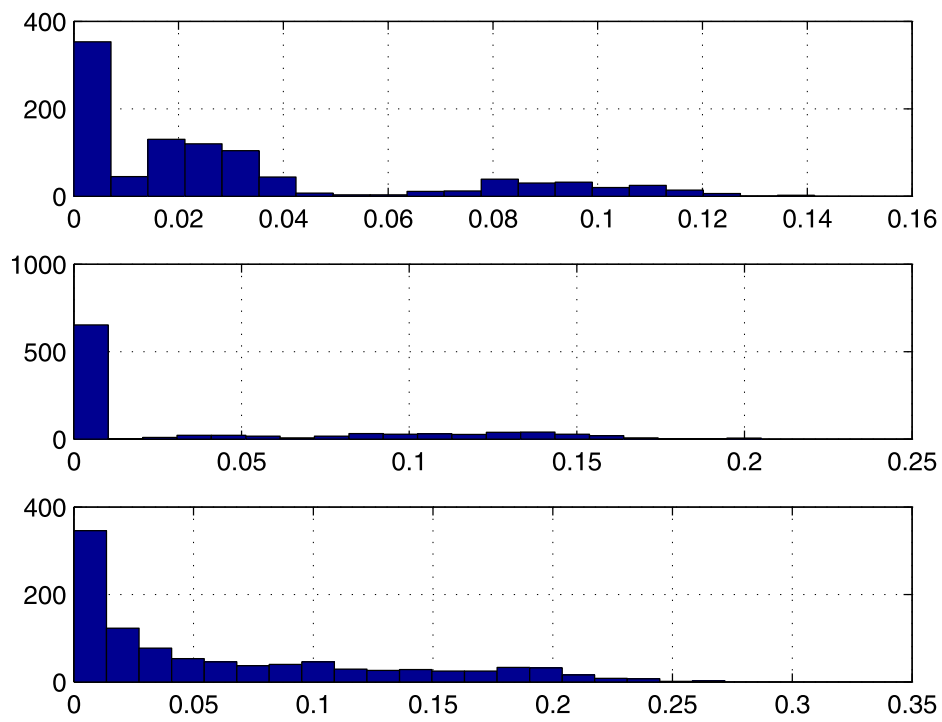

Fig. 6 Examples of a kernel metric histograms

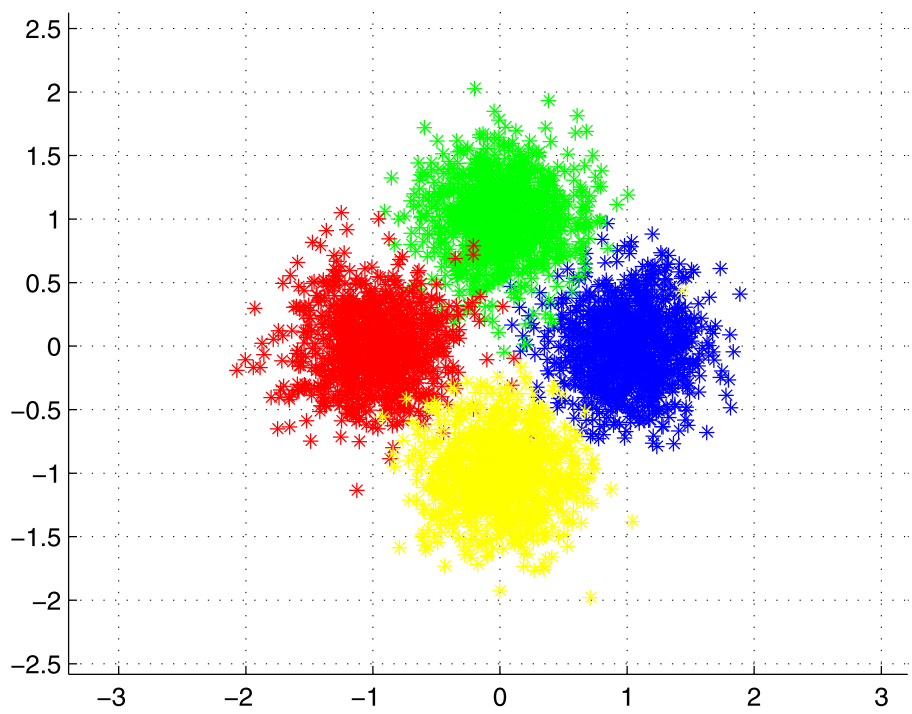

Fig. 7 Four component dataset

\subsection{Gaussian synthetic dataset}

The first dataset contains 4000 items synthesized as a mixture of four two-dimensional Gaussian distributions with independent components having a standard deviation of 0.3 . The means of the distributions are located on the unit circle with the angular distance of $\pi / 2$. A scatter plot of this dataset is presented in Fig. 7. The clusters (components) are overlapped here and a possible answer according to the true number of clusters is obviously 

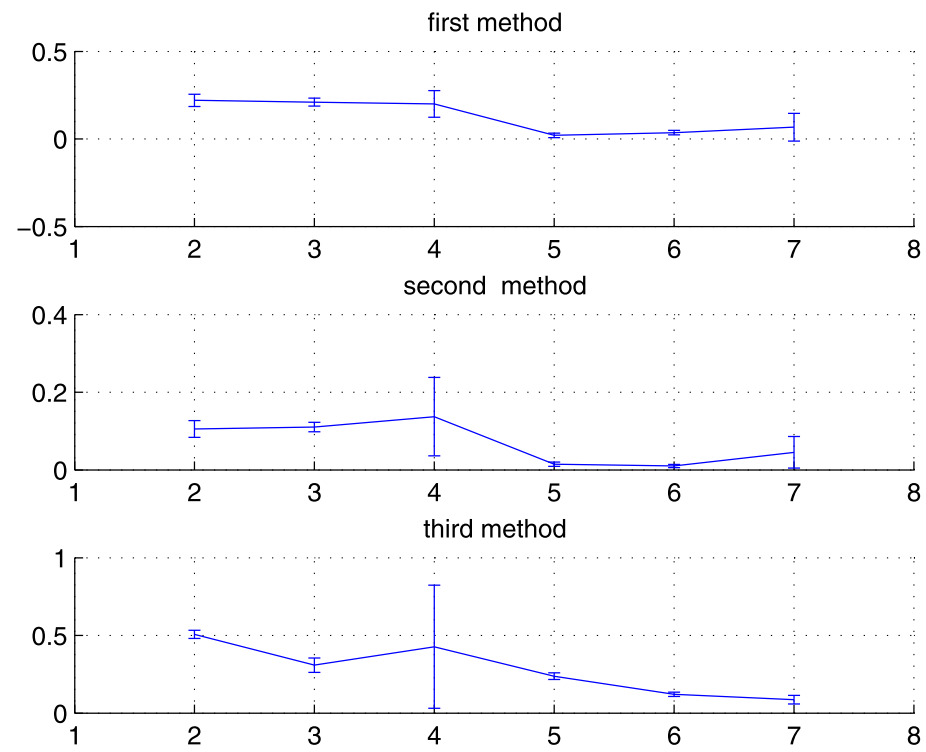

Fig. 8 Error-plots of the lowest groups frequencies for the four component dataset
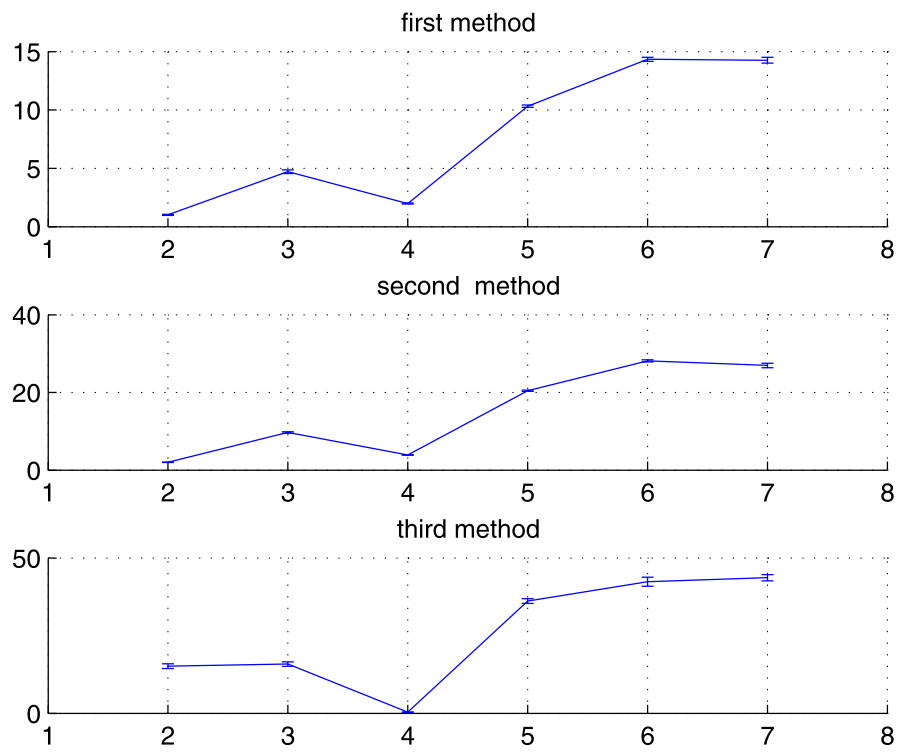

Fig. 9 Error-plots of the normalized proportions' means for the four component dataset

ambiguous to be 2 or 4 . In the experiments with the concentrating measures (2) and (3), 300 pairs of samples having the size of 600 have been selected. Figure 8 demonstrates that the frequency index offers two clusters. The normalized mean index by applying the first and second simulation methods vote also for a two cluster structure while the last simulation method determines four clusters (see, Fig. 9). 

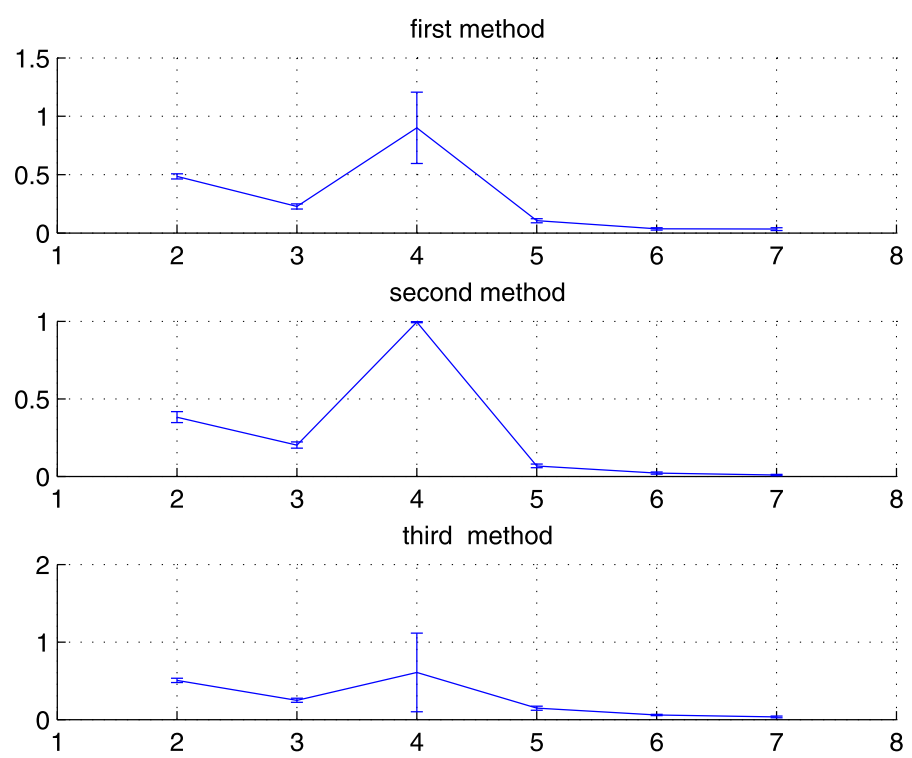

Fig. 10 Error-plots of the lowest groups frequencies for the four component dataset calculated by the first kernel distance

Another situation is observed in the case when a kernel distance is used. The results presented in Figs. 10-15 have been obtained for the kernels:

$-K_{1}(x, y)=\|x-y\|$,

$-K_{2}(x, y)=\exp \left(-\|x-y\|^{2}\right)$,

$-K_{3}(x, y)=\frac{1}{1+\|x-y\|^{2}}$

in the case of 300 samples of size 600 . Here, we also exhibit error-plots bars calculated for the normalized mean value

$$
m e=\frac{m e a n(\text { dist })}{C_{75}(\text { dist })},
$$

where mean(dist) is the distance mean value, and $C_{75}($ dist $)$ is the 75 -th percentile or the third quartile of the distances' values.

All indexes found the "correct" answer with sufficiently high stability rate achieved by the first and the second simulation approaches. It must be also noted that the frequency based index demonstrates more stable behavior.

\subsection{Real datasets}

\subsubsection{A three text collection set}

This dataset is chosen from http://www.dcs.gla.ac.uk/idom/ir_resources/test_collections/ and it consists of the following three text collections

- DC0-Medlars Collection (1033 medical abstracts);

- DC1-CISI Collection (1460 information science abstracts);

- DC2-Cranfield Collection (1400 aerodynamics abstracts). 

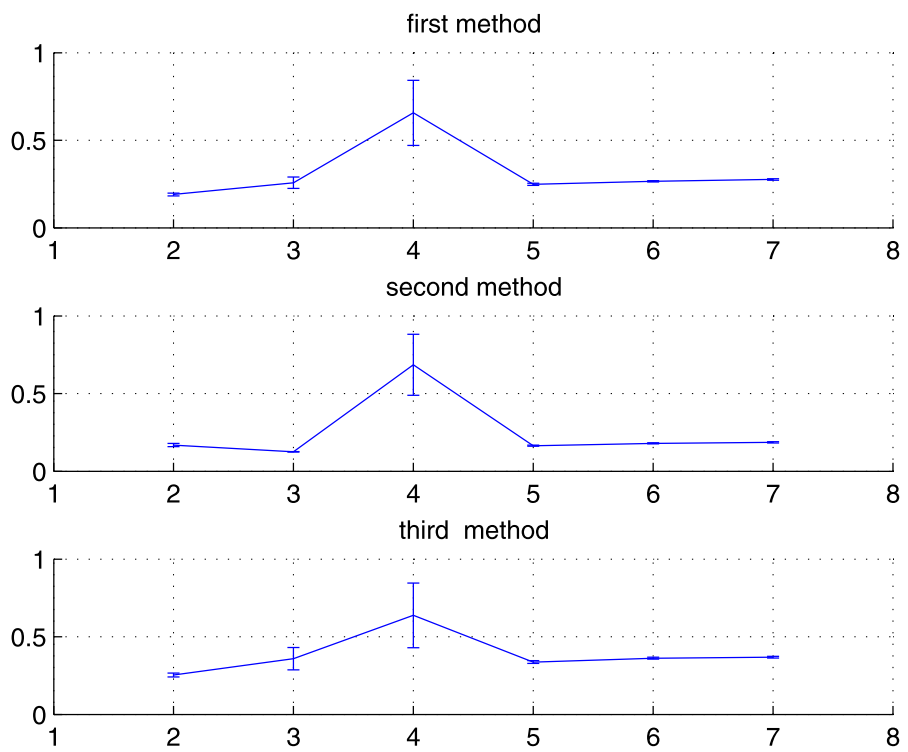

Fig. 11 Error-plots of the normalized mean value for the four component dataset calculated by the first kernel distance
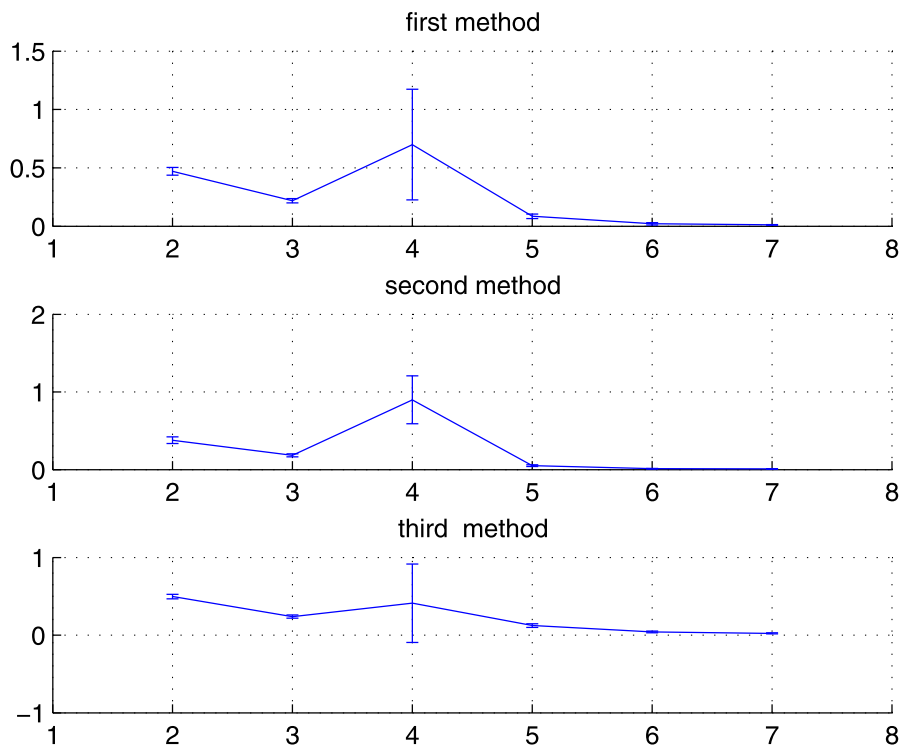

Fig. 12 Error-plots of the lowest groups frequencies for the four component dataset calculated by the second kernel distance

The dataset was analyzed in Dhillon and Modha (2001) by means of the spherical $k$ means algorithm and it has also been considered in several works (see, for example, Kogan et al. 2003a, 2003b, 2003c; Volkovich et al. 2004). Following the famous "bag of words" 

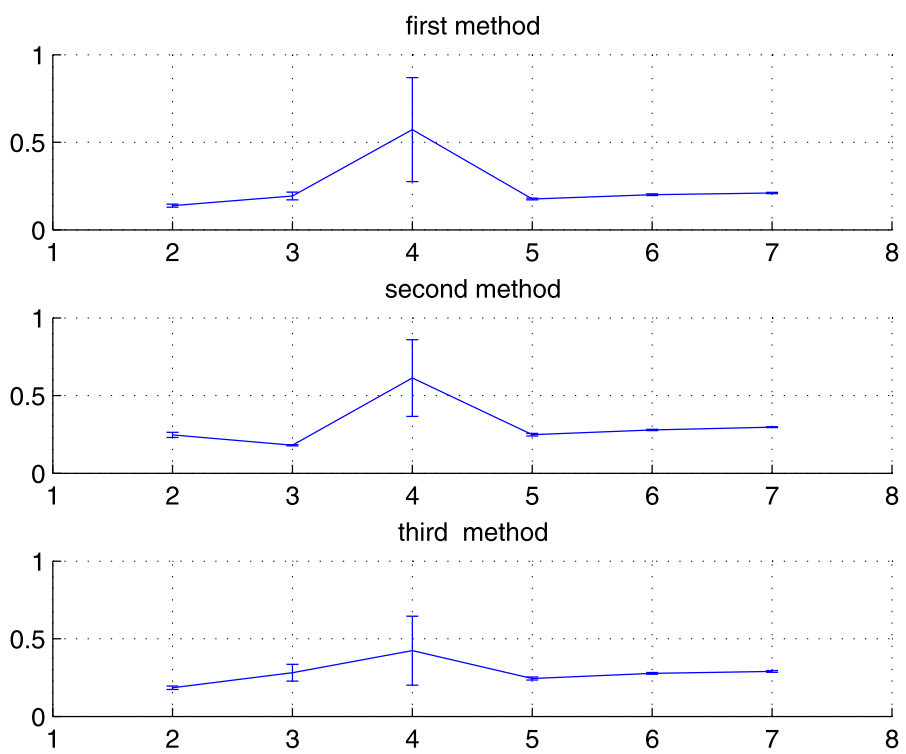

Fig. 13 Error-plots of the normalized mean value for the four component dataset calculated by the second kernel distance
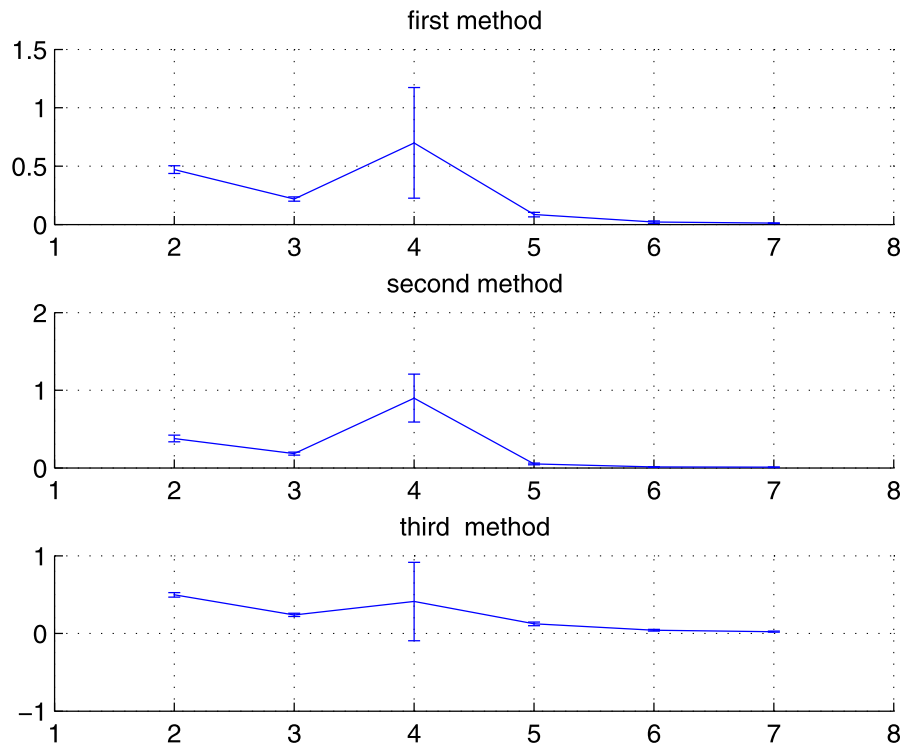

Fig. 14 Error-plots of the lowest groups frequencies for the four component dataset calculated by the third kernel distance

method the 300 "best" terms were selected (see Dhillon et al. 2003 for term selection details) and the dataset is embedded into the real Euclidean space with dimension 300. A dimension reduction is made by the Principal Component Analysis. This dataset is recognized to be 

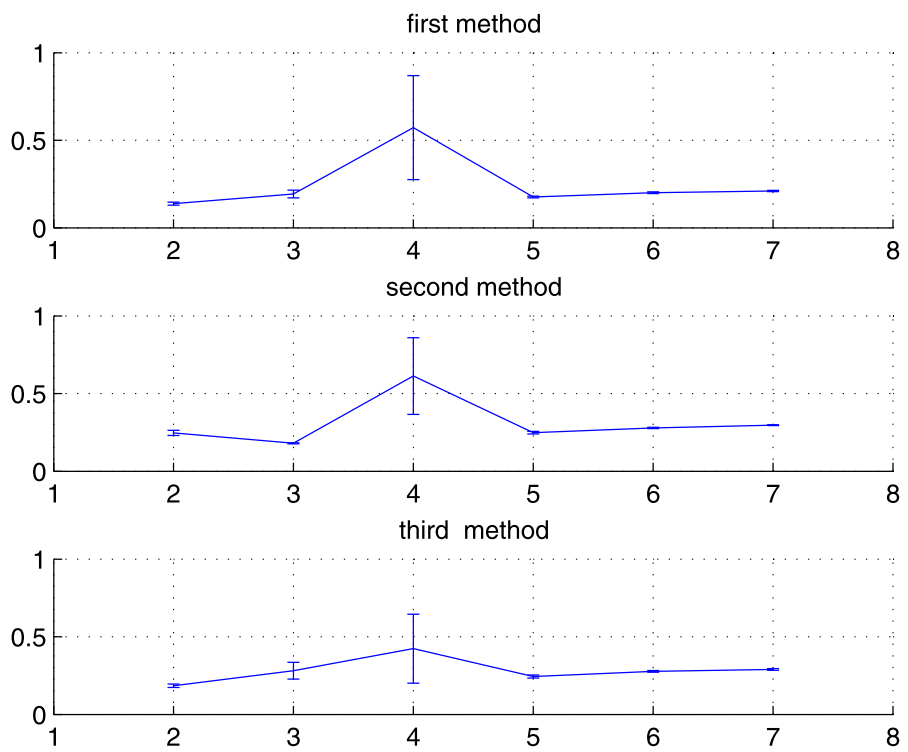

Fig. 15 Error-plots of the normalized mean value for the four component dataset calculated by the third kernel distance
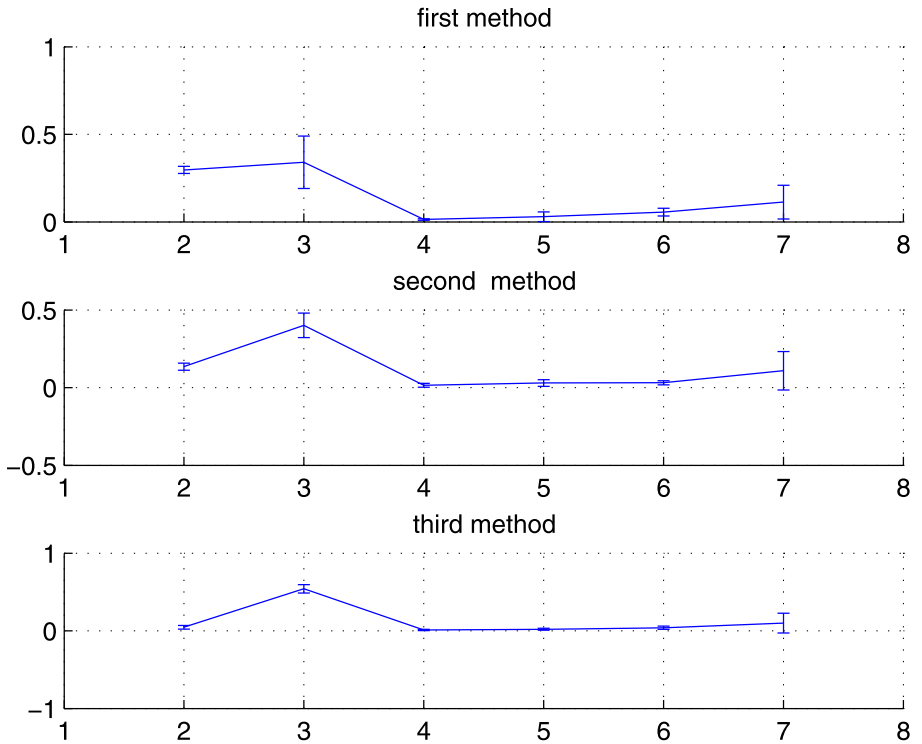

Fig. 16 Error-plots of the lowest groups frequencies for the three text dataset

well separated via the two leading principal components. We employ this representation in our experiments. Outcomes obtained by means of the concentrated measure (2) for 300 sample pairs owing size of 600, are presented in Figs. 16, 17 and 18. 

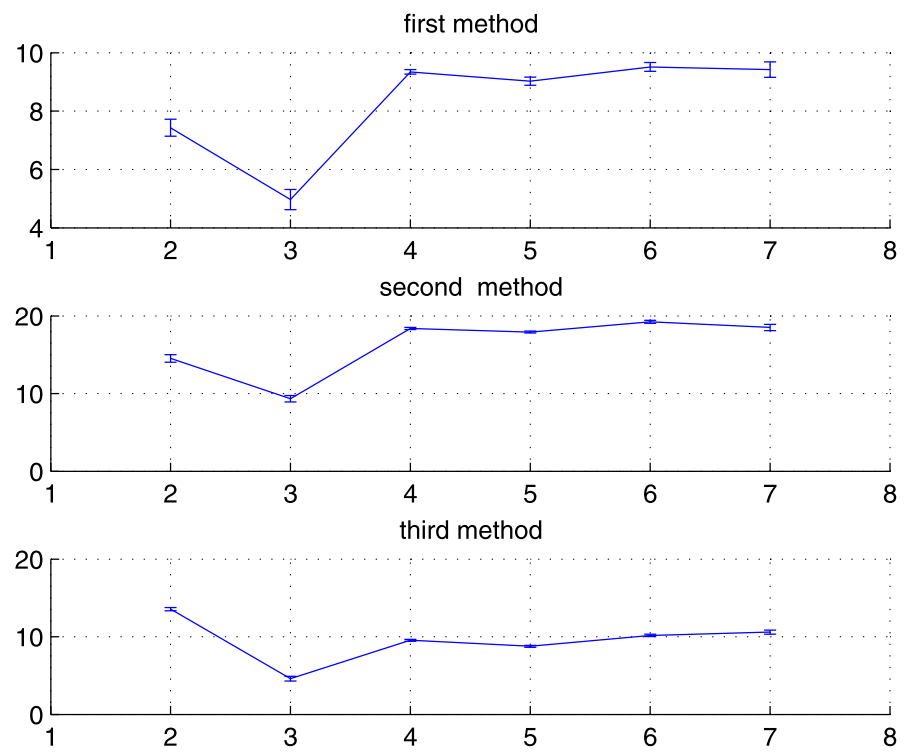

Fig. 17 Error-plots of the normalized proportions' means for the three text dataset
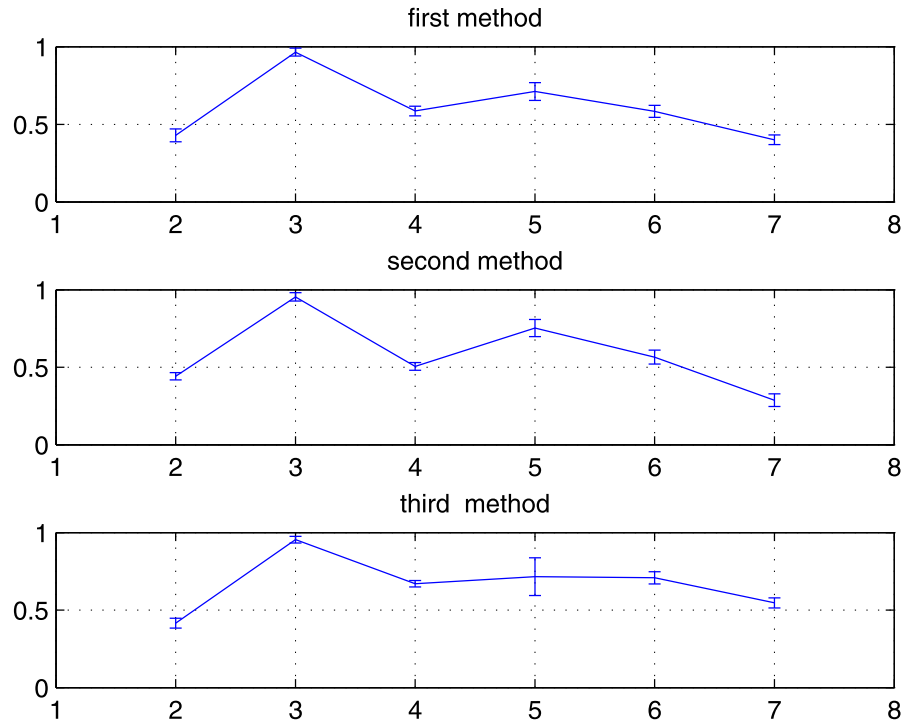

Fig. 18 Error-plots of the lowest groups frequencies for the three texts dataset calculated by the first kernel distance

In all cases, except for the frequency index combined with the first simulation method, the "true" number of clusters has been clearly found. Experiments with kernel distances have been provided for the kernels mentioned in the previous dataset example. 

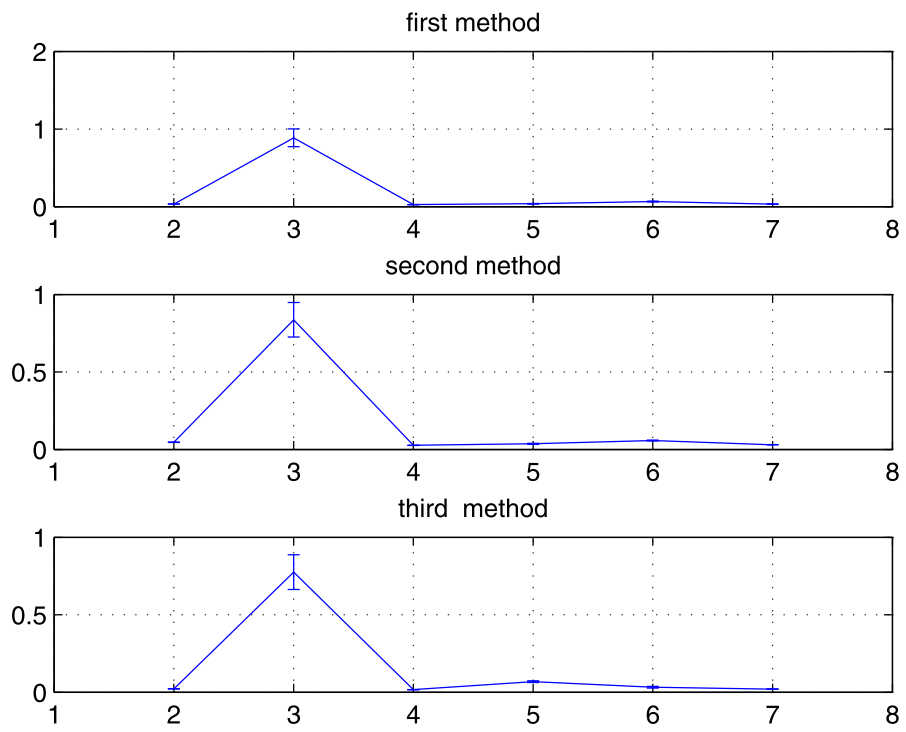

Fig. 19 Error-plots of the normalized means for the three texts dataset calculated by the first kernel distance
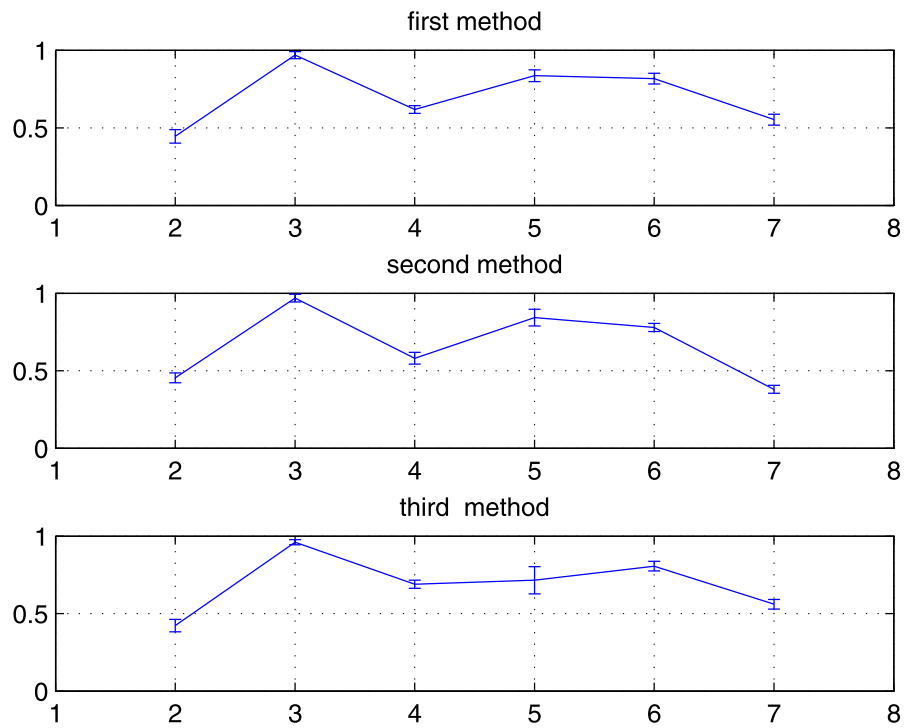

Fig. 20 Error-plots of the lowest groups frequencies for the three texts dataset calculated by the second kernel distance

The results exhibit (see Figs. 19, 20, 21, 22, and 23) similar behavior of the simulations methods which can be recognized as more stable in comparison with the cluster contents based methods, presented in Figs. 16, 17 and 18. 

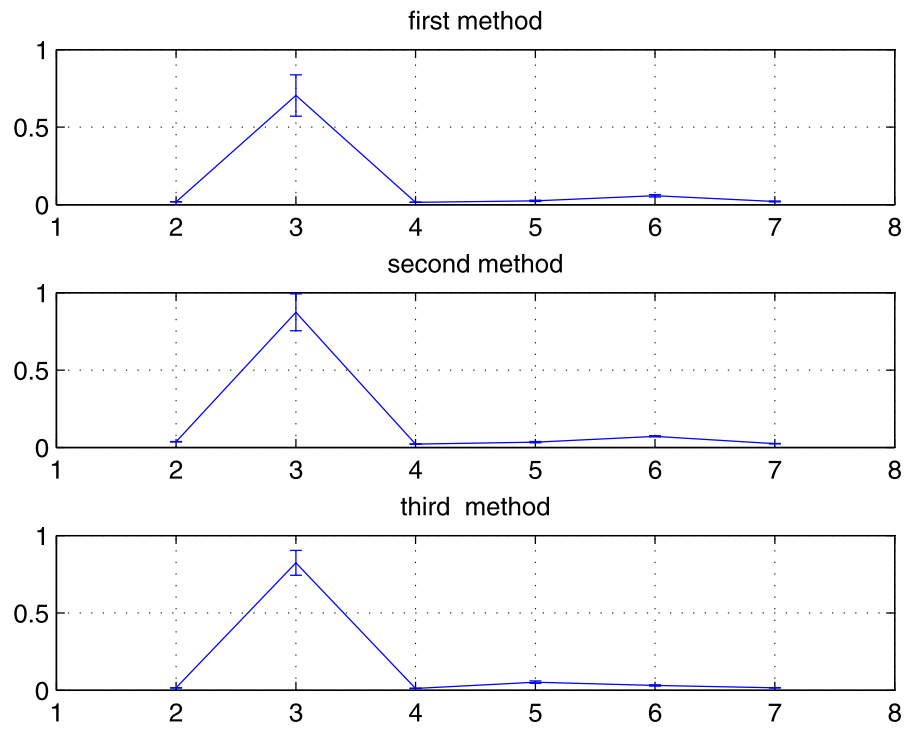

Fig. 21 Error-plots of the normalized means for the three texts dataset calculated by the second kernel distance
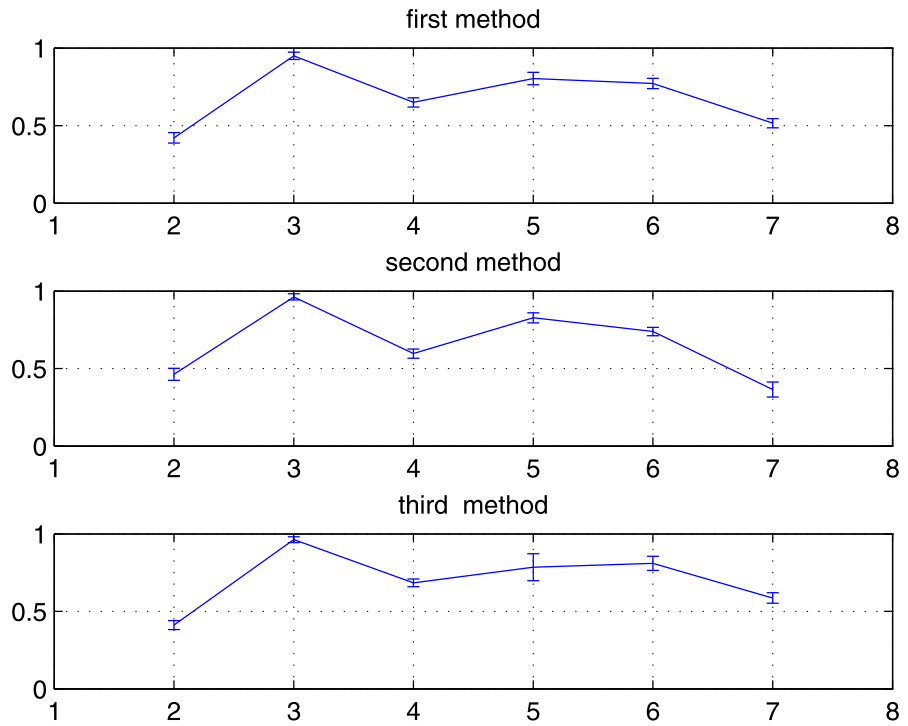

Fig. 22 Error-plots of the lowest groups frequencies for the three texts dataset calculated by the third kernel distance

\subsubsection{Iris flower dataset}

An additional real dataset, which was analyzed, is the well-known Iris flower dataset or Fisher's Iris dataset available, for example, at http://archive.ics.uci.edu/ml/datasets/Iris. 

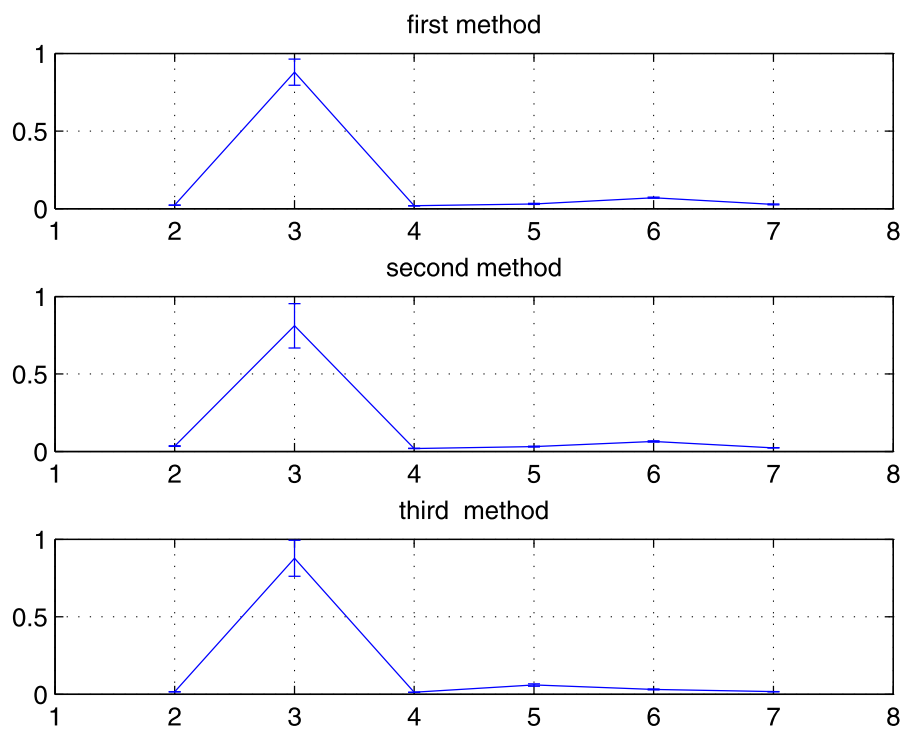

Fig. 23 Error-plots of the normalized means for the three texts dataset calculated by the third kernel distance

The dataset includes 50 samples from each of three species of Iris flowers: I. setosa, I. virginica and I. versicolor. These species belong to three clusters such that one cluster is linearly separable from the others, although the other two are not. This dataset was considered in many papers. For instance, a two cluster structure was detected in Roth et al. (2002). In Figs. 24, 25, 26, and 27, we present results obtained by cluster contents based methods and the first kernel distance. Here, 200 pairs of samples having size 70 were drawn. The third simulation method was not applied due to dataset size limitation. As can be seen, the frequency based indexes and the normalized proportions' means succeed to find the "true" number of clusters. However, the normalized means for the Iris dataset, calculated by the first kernel distance fail. Generally speaking, this approach appears to be less stable compared to earlier examples of big datasets. Apparently, kernel based distances seem to better suit partitions owning sufficiently large clusters.

\subsubsection{A comparison with other methods}

To evaluate the superiority of the proposed methodology we suggest a comparison of its results to outcomes received for the same datasets by means of several other cluster validation approaches. In Table 1 we exhibit the results obtained by the Calinski and Harabasz index ( $C H$-index) (Calinski and Harabasz 1974), the Hartigan index ( $H$-index) (Hartigan 1985), the Krzanowski and Lai index (KL-index) (Krzanowski and Lai 1985), the Sugar and James index ( $S J$-index) (Sugar and James 2003) and the GAP-index of Tibshirani et al. (2001). The analyzed datasets are denoted by $G-4-03, D-3-300$ and Iris, correspondingly, to the order of their appearance in this section. Our method, designated as Kern_Based method, performs properly in the comparison, since it is the only one which fits the true number of clusters for all datasets.

The H-method gives a wrong answer only for the Iris dataset. As mentioned earlier, the clusters' structure is configured here in the way that one group is linearly separable from the others, while the other two are not. Thus, a two-clusters solution can be plausible (see, 

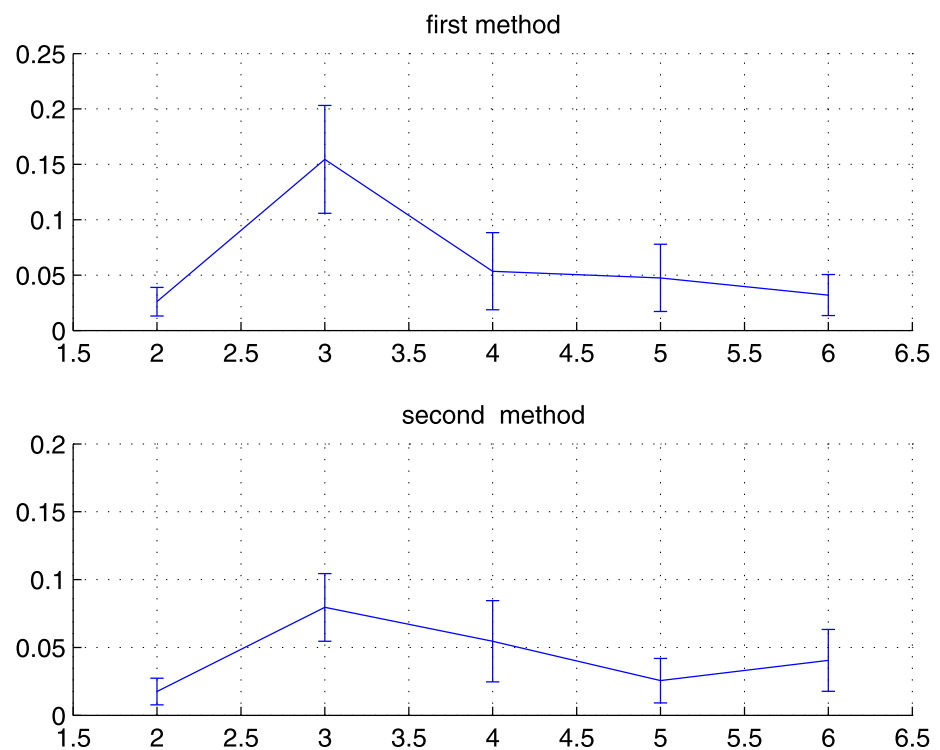

Fig. 24 Error-plots of the lowest groups frequencies for the Iris dataset
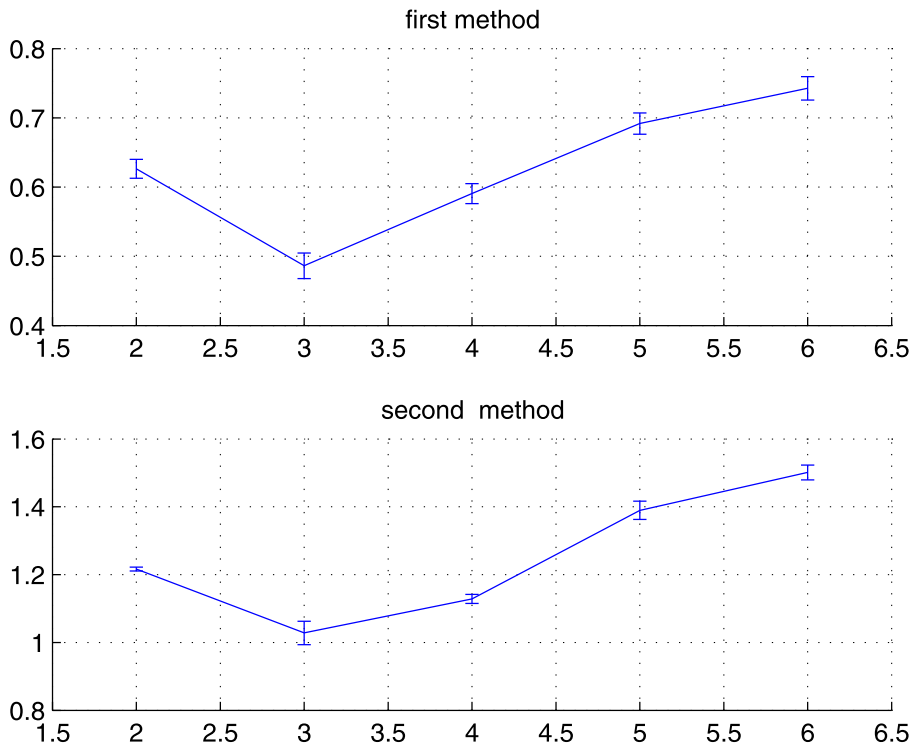

Fig. 25 Error-plots of the normalized proportions' means for the Iris dataset

for example, Roth et al. 2002). It can be suggested that the Gap method fails as well on this dataset due to the same reason. In the case of the four-components dataset this approach errs in the light of a significant clusters' intersection. 

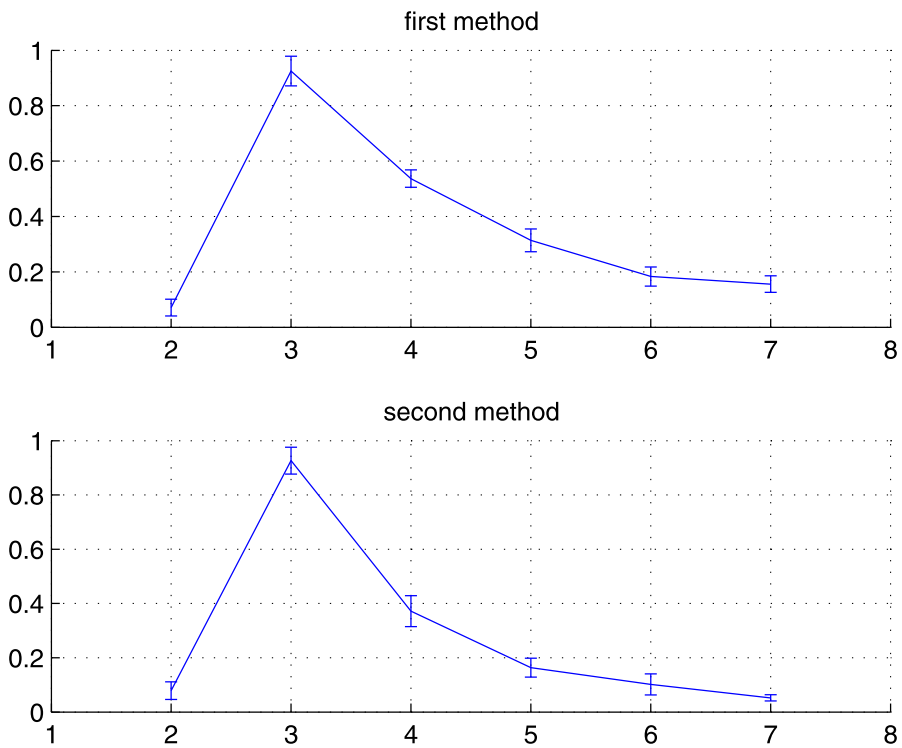

Fig. 26 Error-plots of the lowest groups frequencies for the Iris dataset calculated by the first kernel distance

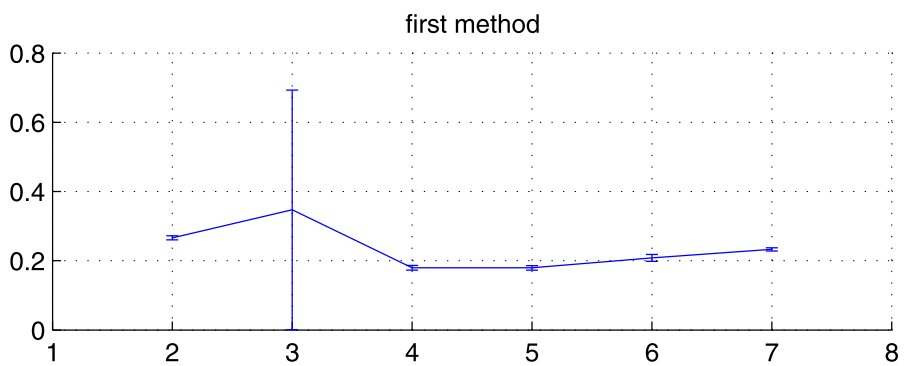

second method

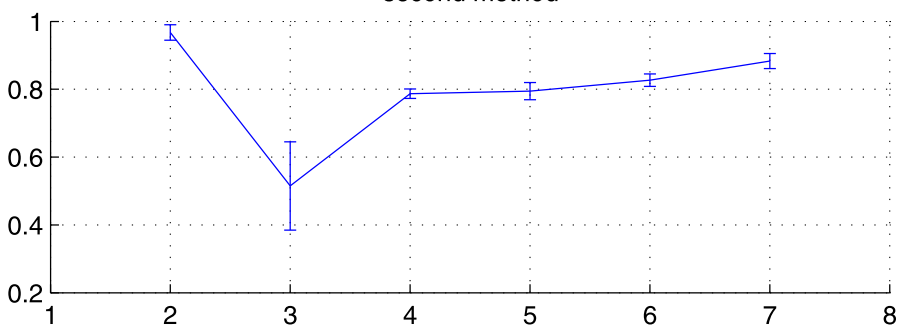

Fig. 27 Error-plots of the normalized proportions' means for the Iris dataset calculated by the first kernel distance

\section{Conclusion}

We propose a new method for detecting the true number of clusters by the stability methodology. In this study we combine several known and sound statistical and probabilistic meth- 
Table 1 Number of clusters obtained by various methods

\begin{tabular}{llll}
\hline & $G-4-03$ & Iris & $D-3-300$ \\
\hline CH-index & 4 & 3 & 6 \\
H-index & 4 & 2 & 3 \\
KL-index & 5 & 2 & - \\
SJ-index & 5 & 3 & - \\
GAP-index & 3 & 3,6 & 2 \\
Kern_Based & 4 & 3 & 3 \\
True & 4 & 3 & 3 \\
\hline
\end{tabular}

ods for the creation of new approaches for the characterizations of clusters structures. Each clustered partition leads to the mixed decomposition of the underlying distribution. A clustering algorithm is an important ingredient of the approach because partitions constructed by means of the algorithm are intended to reflect the inner hidden data structure, corresponding to the underlying mixture distributions. In the current paper we apply a popular clustering algorithm-the $k$-means. In addition to a clustering algorithm, there are two additional crucial components of the methodology: the first one is a probability distance anticipated to measure proximity of random variables, and an approach proposed to simulate samples occurrences in clusters. We discuss the probability metrics notion and present several important approaches which are highlighted. Here, we especially pay our attention to the positive and the negative definite kernel based distances. The simulation phase creates samples in the clusters which are representing independent random variables. This task addresses the problem related to the comparison between two solutions obtained from different sets. In the current paper we develop three procedures for obtaining these results. To demonstrate the effectiveness of the proposed procedures, we present several examples in which the true number of clusters is looked for.

\section{References}

Aizerman, M. A., Braverman, E. M., \& Rozono, L. I. (1964). Theoretical foundations of the potential function method in pattern recognition learning. Automation and Remote Control, 25, 821-837.

Anderson, N. H., Hall, P., \& Titterington, M. (1994). Two-sample test statistics for measuring discrepancies between two multivariate probability density functions using kernel-based density estimates. Journal of Multivariate Analysis, 50(1), 41-54.

Aronszajn, N. (1950). Theory of reproducing kernels. Transactions of the American Mathematical Society, 68.

Baringhaus, L., \& Franz, C. (2004). On a new multivariate two-sample test. Journal of Multivariate Analysis, 88(1), 190-206.

Barzily, Z., Volkovich, Z., Akteke-Ozturk, B., \& Weber, G.-W. (2009). On a minimal spanning tree approach in the cluster validation problem. Informatica, 20(2), 187-202.

Belopolskaya, Ya., Klebanov, L., \& Volkovich, V. (2005). Characterization of elliptic distributions. Journal of Mathematical Sciences, 127(1), 1682-1686.

Ben-Hur, A., \& Guyon, I. (2003). Detecting stable clusters using principal component analysis. In M. J. Brownstein \& A. Khodursky (Eds.), Methods in molecular biology (pp. 159-182). Clifton: Humana Press.

Ben-Hur, A., Horn, D., Siegelmann, H. T., \& Vapnik, V. (2001). Support vector clustering. Journal of Machine Learning Research, 2, 125-137.

Ben-Hur, A., Elisseeff, A., \& Guyon, I. (2002). A stability based method for discovering structure in clustered data. In Pacific symposium on biocomputing (pp. 6-17).

Berg, C., Christensen, J. P. R., \& Ressel, P. (1984). Harmonic analysis on semigroups. Berlin: Springer.

Breckenridge, J. (1989). Replicating cluster analysis: method, consistency and validity. Multivariate Behavioral Research, 24, 147-161. 
Calinski, R., \& Harabasz, J. (1974). A dendrite method for cluster analysis. Communications in Statistics, 3, $1-27$.

Celeux, G., \& Govaert, G. (1992). A classification EM algorithm for clustering and two stochastic versions. Computational Statistics \& Data Analysis, 14(3), 15, 315-332.

Chakravarthy, S. V., \& Ghosh, J. (1996). Scale-based clustering using the radial basis function network. IEEE Transactions on Neural Networks, 7(5), 1250-1261.

Cheng, R., \& Milligan, G. W. (1996). Measuring the influence of individual data points in a cluster analysis. Journal of Classification, 13, 315-335.

Conover, W. J., Johnson, M. E., \& Johnson, M. M. (1981). Comparative study of tests of homogeneity of variances, with applications to the outer continental shelf bidding data. Technometrics, 23, 351-361.

Cortes, C., \& Vapnik, V. (1995). Support-vector networks. Machine Learning, 273-297.

Cuevas, A., Febrero, M., \& Fraiman, R. (2000). Estimating the number of clusters. Canadian Journal of Statistics, 28(2), 367-382.

Cuevas, A., Febrero, M., \& Fraiman, R. (2001). Cluster analysis: a further approach based on density estimation. Computational Statistics \& Data Analysis, 28, 441-459.

Dhillon, I. S., \& Modha, D. S. (2001). Concept decompositions for large sparse text data using clustering. Machine Learning, 42(1), 143-175. Also appears as IBM Research Report RJ 10147, July 1999.

Dhillon, I., Kogan, J., \& Nicholas, C. (2003). Feature selection and document clustering. In A comprehensive survey of text mining (pp. 73-100). Berlin: Springer.

Dudoit, S., \& Fridlyand, J. (2002). A prediction-based resampling method for estimating the number of clusters in a dataset. Genome Biology, 3(7), 0036.

Dunn, J. C. (1974). Well separated clusters and optimal fuzzy partitions. Journal of Cybernetics, 4, 95-104.

Duran, B. S. (1976). A survey of nonparametric tests for scale. Communications in Statistics. Theory and Methods, 5, 1287-1312.

Feng, Y., \& Hamerly, G. (2006). PG-means: learning the number of clusters in data. In Proceedings of the twentieth annual conference on neural information processing systems (NIPS).

Filippone, M., Camastra, F., Masulli, F., \& Rovetta, S. (2008). A survey of kernel and spectral methods for clustering. Pattern Recognition, 41(1), 176-190.

Friedman, J. H., \& Rafsky, L. C. (1979). Multivariate generalizations of the Wolfowitz and Smirnov twosample tests. Annals of Statistics, 7, 697-717.

Girolami, M. (2002). Mercer kernel-based clustering in feature space. IEEE Transactions on Neural Networks, 13(3), 780-784.

Gokcay, E., \& Principe, J. C. (2002). Information theoretic clustering. IEEE Transactions on Pattern Analysis and Machine Intelligence, 24(2), 158-171.

Gordon, A. D. (1994). Identifying genuine clusters in a classification. Computational Statistics \& Data Analysis, 18, 561-581.

Gordon, A. D. (1999). Classification. Boca Raton: Chapman and Hall/CRC.

Gretton, A., Borgwardt, K., Rasch, M., Schölkopf, B., \& Smola, A. (2007a). A kernel method for the twosample-problem. In Advances in neural information processing systems (Vol. 19, pp. 513-520). Cambridge: MIT Press.

Gretton, A., Borgwardt, K., Rasch, M., Schölkopf, B., \& Smola, A. (2007b). A kernel approach to comparing distributions. In Proceedings of the 22nd conference on artificial intelligence (AAAI-07) (pp. 16371641).

Gretton, A., Borgwardt, K. M., Rasch, M. J., Schölkopf, B., \& Smola, A. J. (2008a). A kernel method for the two-sample problem. CoRR. arXiv:0805.2368. DBLP, http://dblp.uni-trier.de.

Gretton, A., Borgwardt, K. M., Rasch, M. J., Schölkopf, B., \& Smola, A. (2008b). A kernel method for the two-sample problem. Journal of Machine Learning Research, 4, 1-10.

Hall, P., \& Tajvidi, N. (2002). Permutation tests for equality of distributions in high-dimensional settings. Biometrika, 89(2), 359-374.

Hamerly, G., \& Elkan, Ch. (2003). Learning the $k$ in $k$-means. In Proceedings of the seventeenth annual conference on neural information processing systems (NIPS) (pp. 281-288).

Hartigan, J. A. (1975). Clustering algorithms. New York: Wiley.

Hartigan, J. A. (1981). Consistency of single linkage for high-density clusters. Journal of the American Statistical Association, 76, 388-394.

Hartigan, J. A. (1985). Statistical theory in clustering. Journal of Classification, 2, 63-76.

Haussler, D. (1999). Convolution kernels on discrete structures (UCSC-CRL-9910). Department of Computer Science University of California at Santa Cruz.

Henze, N. (1988). A multivariate two-sample test based on the number of nearest neighbor type coincidences. Annals of Statistics, 16, 772-783.

Hubert, L., \& Schultz, J. (1976). Quadratic assignment as a general data-analysis strategy. British Journal of Mathematical \& Statistical Psychology, 76, 190-241. 
Jain, A., \& Dubes, R. (1988). Algorithms for clustering data. New Jersey: Englewood Cliffs/Prentice-Hall.

Jain, A. K., \& Moreau, J. V. (1987). Bootstrap technique in cluster analysis. Pattern Recognition, 20(5), $547-568$.

Kass, R. E. (1995). A reference Bayesian test for nested hypotheses and its relationship to the Schwarz criterion. Journal of the American Statistical Association, 90, 928-934.

Kaufman, L., \& Rousseeuw, P. J. (1990). Finding groups in data. New York: Wiley.

Klebanov, L. (2003). One class of distribution free multivariate tests. SPb. Math. Society, Preprint, 03.

Klebanov, L. B. (2005). $N$-distances and their applications. Charsel University in Prague, The Karolinum Press.

Klebanov, L., Kozubowskii, T., Rachev, S., \& Volkovich, V. (2001). Characterization of distributions symmetric with respect to a group of transformations and testing of corresponding statistical hypothesis. Statistics \& Probability Letters, 53, 241-247.

Kogan, J., Nicholas, C., \& Volkovich, V. (2003a). Text mining with information-theoretical clustering. Computing in Science and Engineering, 52-59.

Kogan, J., Nicholas, C., \& Volkovich, V. (2003b). Text mining with hybrid clustering schemes. In M. W. Berry \& W. M. Pottenger (Eds.), Proceedings of the workshop on text mining (pp. 5-16). Held in conjunction with the third SIAM international conference on data mining.

Kogan, J., Teboulle, M., \& Nicholas, C. (2003c). Optimization approach to generating families of $k$-means like algorithms. In Proceedings of the workshop on clustering high dimensional data and its applications. Held in conjunction with the third SIAM international conference on data mining.

Krzanowski, W., \& Lai, Y. (1985). A criterion for determining the number of groups in a dataset using sum of squares clustering. Biometrics, 44, 23-34.

Kuhn, H. (1955). The Hungarian method for the assignment problem. Naval Research Logistics Quarterly, 2, 83-97.

Lange, T., Braun, M., Roth, V., \& Buhmann, J. M. (2003). Stability-based model selection. Advances in Neural Information Processing Systems, 15. http://citeseer.ist.psu.edu/700728.html.

Lange, T., Roth, V., Braun, M., \& Buhmann, J. M. (2004). Stability-based validation of clustering solutions. Neural Computation, 15(6), 1299-1323.

Levine, E., \& Domany, E. (2001). Resampling method for unsupervised estimation of cluster validity. Neural Computation, 13, 2573-2593.

Lukacs, E. (1970). Characteristic functions. Duxbury: Griffin.

Milligan, G., \& Cooper, M. (1985). An examination of procedures for determining the number of clusters in a data set. Psychometrika, 50, 159-179.

Mufti, G. B., Bertrand, P., \& El Moubarki, L. (2005). Determining the number of groups from measures of cluster validity. In Proceedings of ASMDA 2005 (pp. 404-414).

Parzen, E. (1962). On the estimation of a probability density function and the mode. Annals of Mathematical Statistics, 32, 1065-1076.

Pascual, D., Pla, F., \& Sanche, J. S. (2010). Cluster validation using information stability measures. Pattern Recognition Letters, 31, 454-461.

Pelleg, D., \& Moore, A. (2000). X-means: Extending K-means with efficient estimation of the number of clusters. In Proceedings of the 17th international conf. on machine learning (pp. 727-734). San Francisco: Morgan Kaufmann.

Principe, J., Xu, D., \& Fisher, J. (2002). Information theoretic learning. In Unsupervised adaptive filtering, I (pp. 265-319). New York: Wiley.

Rachev, S. T. (1991). Wiley series in probability and mathematical statistics. Probability metrics and the stability of stochastic models. Chichester: Wiley.

Rose, K., Gurewitz, E., \& Fox, G. C. (1990). Statistical mechanics and phase transitions in clustering. Physical Review Letters, 65(8), 945-948.

Rose, K., Gurewitz, E., \& Fox, G. C. (1993). Constrained clustering as an optimization method. IEEE Transactions on Pattern Analysis and Machine Intelligence, 15(8), 785-794.

Robert, J., \& Torbjorn, E. (2008). A new information theoretic analysis of sum-of-squared-error kernel clustering. Neurocomputing, 72(1-3), 23-31.

Rosenbaum, P. (2005). An exact distribution-free test comparing two multivariate distributions based on adjacency. Journal of the Royal Statistical Society. Series B, Statistical Methodology, 67(4), 515-530.

Roth, V., Lange, T., Braun, M., \& Buhmann, J. (2002). A resampling approach to cluster validation. COMPSTAT, available at http://www.cs.uni-bonn.De/braunm.

Sato, K. (1999). Levy processes and infinitely divisible distributions. Cambridge: Cambridge University Press.

Schoenberg, I. J. (1938). Metric spaces and positive definite functions. Transactions of the American Mathematical Society, 44(3), 522-536.

Schölkopf, B. (2000). The kernel trick for distances. In NIPS (pp. 301-307). 
Schölkopf, B., \& Smola, A. J. (2002). Learning with kernels. New York: MIT Press.

Schölkopf, B., Smola, A. J., \& Muller, K.-R. (1998). Nonlinear component analysis as a kernel eigenvalue problem. Neural Computation, 10(5), 1299-1319.

Steinwart, I. (2001). On the influence of the kernel on the consistency of support vector machines. Journal of Machine Learning Research, 2, 67-93.

Still, S., \& Bialek, W. (2004). How many clusters? An information-theoretic perspective. Neural Computation, 16(12), 2483-2506.

Strehl, A., \& Ghosh, J. (2002). Cluster ensembles - a knowledge reuse framework for combining multiple partitions. Journal of Machine Learning Research, 3, 583-617.

Stuetzle, W. (2003). Estimating the cluster tree of a density by analyzing the minimal spanning tree of a sample. Journal of Classification, 20(5), 25-47.

Sugar, C., \& James, G. (2003). Finding the number of clusters in a data set: an information theoretic approach. Journal of the American Statistical Association, 98, 750-763.

Tibshirani, R., \& Walther, G. (2005). Cluster validation by prediction strength. Journal of Computational and Graphical Statistics, 14(3), 511-528.

Tibshirani, R., Walther, G., \& Hastie, T. (2001). Estimating the number of clusters via the gap statistic. Journal of the Royal Statistical Society. Series B, Statistical Methodology, 63(2), 411-423.

Tishby, N., Pereira, F. C., \& Bialek, W. (2000). The information bottleneck method. In B. Hajek \& R. S. Sreenivas (Eds.), Proceedings of the 37th annual Allerton conference on communication, control and computing (pp. 368-377).

Volkovich, Z., \& Barzily, Z. (2007). On application of probability metrics in the cluster stability problem. In 1st European conference on data mining (ECDM'07) (pp. 5-7). Lisbon, Portugal.

Volkovich, V., Kogan, J., \& Nicholas, C. (2004). $k$-means initialization by sampling large datasets. In I. Dhillon \& J. Kogan (Eds.), Proceedings of the workshop on clustering high dimensional data and its applications (pp. 17-22). Held in conjunction with SDM 2004.

Volkovich, Z., Barzily, Z., \& Sureanu, P. (2005). The Levy-Khinchine representations and functional algebras of test functions. Journal of Pure and Applied Mathematics, 25(1), 103-121.

Volkovich, Z., Barzily, Z., \& Morozensky, L. (2008). A statistical model of cluster stability. Pattern Recognition, 41(7), 2174-2188.

Volkovich, Z., Barzily, Z., Avros, R., \& Toledano-Kitai, D. (2009a). On application of the $K$-nearest neighbors approach for cluster validation. In Proceeding of the XIII international conference applied stochastic models and data analysis (ASMDA 2009). Vilnius, Lithuania

Volkovich, Z., Barzily, Z., Weber, G.-W., \& Toledano-Kitai, D. (2009b). Cluster stability estimation based on a minimal spanning trees approach. In The second global conference on power and optimization (PCO 2009). Bali, Indonesia.

Volkovich, Z., Weber, G.-W., \& Avros, R. (2010). On an adjacency cluster merit. In The third global conference on power control and optimization (PCO 2010). Gold Coast, Australia.

Wand, M. P., \& Jones, M. C. (1995). Kernel smoothing. London: Chapman and Hall.

Wishart, D. (1969). Mode analysis: a generalization of nearest neighbor which reduces chaining effects. In A. J. Cole (Ed.), Numerical taxonomy (Vol. 76, pp. 282-311). London: Academic Press.

Zech, G., \& Aslan, B. (2005). New test for the multivariate two-sample problem based on the concept of minimum energy. Journal of Statistical Computation and Simulation, 75(2), 109-119.

Zinger, A. A., Kakosyan, A. V., \& Klebanov, L. B. (1989). Characterization of distributions by means of the mean values of statistics in connection with some probability metrics. Stability Problems for Stochastic Models, VNIISI, 47-55.

Zolotarev, V. M. (1997). Modern theory of summation of random variable. Leiden: Brill Academic. 